University of Rhode Island

DigitalCommons@URI

Open Access Dissertations

2019

\title{
LASER-ASSISTED TRANSDERMAL DRUG DELIVERY AND VACCINATION
}

Prateek Kakar

University of Rhode Island, prateek_kakar@uri.edu

Follow this and additional works at: https://digitalcommons.uri.edu/oa_diss

\section{Recommended Citation}

Kakar, Prateek, "LASER-ASSISTED TRANSDERMAL DRUG DELIVERY AND VACCINATION" (2019). Open Access Dissertations. Paper 859.

https://digitalcommons.uri.edu/oa_diss/859

This Dissertation is brought to you for free and open access by DigitalCommons@URI. It has been accepted for inclusion in Open Access Dissertations by an authorized administrator of DigitalCommons@URI. For more information, please contact digitalcommons-group@uri.edu. 


\section{LASER-ASSISTED TRANSDERMAL DRUG DELIVERY}

AND VACCINATION

BY

PRATEEK KAKAR

A DISSERTATION SUBMITTED IN PARTIAL FULFILLMENT OF THE

REQUIREMENTS FOR THE DEGREE OF

DOCTOR OF PHILOSOPHY

IN

PHARMACEUTICAL SCIENCES

UNIVERSITY OF RHODE ISLAND 


\section{DOCTOR OF PHILOSOPHY DISSERTATION}

OF

\section{PRATEEK KAKAR}

\section{APPROVED:}

Dissertation Committee:

Major Professor Xinyuan Chen

Fatemeh Akhlaghi

Yi Zheng

Nasser H. Zawia

DEAN OF THE GRADUATE SCHOOL

\section{UNIVERSITY OF RHODE ISLAND}

2019 


\begin{abstract}
Oral drug delivery has been the major route of drug delivery due to the ease of drug administration and patient compliance. Transdermal drug delivery is highly attractive for alternative drug delivery and yet faces significant challenges, largely due to the barrier function of the Stratum Corneum (SC) layer of the skin. Different chemical and physical methods have been explored to disrupt the SC layer to facilitate transdermal drug delivery. However, those methods only received limited successes due to the low efficiency in SC disruption or the potential safety risks.

Ablative fractional laser (AFL) emerged in the last 2 decades as a novel technology for safe and efficient ablation of SC layer to facilitate transdermal drug delivery. AFL was fabricated based on the concept of fractional photothermolysis for improved skin resurfacing in cosmetic field. This technology emits tiny laser beams on skin surface to instantly increase skin temperature to over $100^{\circ} \mathrm{C}$ and cause tissue evaporation. Because of this, tiny microchannels (MCs) are generated on skin surface to allow drugs to migrate into the skin with high efficiency. At the same time, the MCs can achieve quick and complete skin recovery in 2-3 days considering each of these MCs is surrounded by normal healthy skin with a good repairing capacity. AFL adequately addresses the dilemma about the safety and efficiency of SC ablation to facilitate transdermal drug delivery. The current dissertation focuses on developing novel delivery platforms for use in conjunction with AFL for extended drug release (Manuscript I and III) and transcutaneous vaccination (Manuscript II). In manuscript I, we coated 'bulk' amount of drug powder into reservoir patches and then topically applied onto AFL-treated skin to elicit multi-day sustained drug release.
\end{abstract}


We found tapped coating could elicit 3-day sustained drug release, while compression coating could elicit 6-day sustained drug release. Drugs in gram scales can be potentially delivered via AFL-assisted reservoir patch delivery with compression coating. We further found such a delivery platform is suitable for the delivery of both small chemicals and macromolecules.

In manuscript II, we coated vaccine powder on full-surface adhesive patches followed by topical application onto AFL-treated skin for transcutaneous vaccination. Beside delivery of vaccines alone, we found a clinical monophosphoryl lipid a (MPL) adjuvant could be encapsulated into poly(lactic-co-glycolic acid) (PLGA) nanoparticles (NPs) for delivery together with vaccines via the same delivery platform. AFL-assisted full-surface powder vaccine/MPL delivery induced much stronger vaccine-specific immune responses than vaccine delivery alone in both newborn and adult mice.

In manuscript III, we embedded drugs into hydrogels for delivery via AFL-generated skin MCs. We found poly (vinyl alcohol) (PVA) hydrogel but not poly (2hydroxyethyl methacrylate) (pHEMA) hydrogel could serve as an efficient delivery platform to deliver drugs via AFL-generated skin MCs. AFL-assisted PVA hydrogelbased zidovudine delivery was found to elicit almost $100 \%$ delivery efficiency and at the same time extend drug release to $\sim 15$ hours, while oral delivery only induced sustained release for up to 3 hours.

AFL-assisted high-dose 6-day sustained powder drug delivery is promising to reduce dosing frequency and improve patient adherence in treatment of long-term chronic diseases. AFL-assisted hydrogel-based drug delivery provides another delivery 
platform for extended delivery of hydrophilic drugs. AFL-assisted full-surface vaccine/MPL delivery represents an innovative transcutaneous vaccination technology for needle-free delivery and adjuvantation of vaccine immunization. The needle-free AFL-based drug/vaccine delivery platform warrants further investigation for human use. 


\section{ACKNOWLEDGMENTS}

I would firstly like to thank my graduate advisor, Dr. Xinyuan Chen for his constant support through these years. I have learnt a lot from him. He motivated me to think critically and come up with innovative and creative ideas. I am also thankful to him for making sure I had all the resources I needed for my research, be it supplies or funding. He provided me with opportunities in all arenas of academic life within the school as well as for external conferences.

I would like to thank my committee members, Dr. Fatemeh Akhlaghi, Dr. Yi Zheng and Dr. Hongyan Yuan for their valuable feedback during committee meetings and comprehensive examinations. They were very flexible with their schedules to make time for all important milestones in my $\mathrm{PhD}$ journey.

I would also like to thank my laboratory members Dr. Nazir Hossen, Dr. Yan Cao, Dr. Xiaoyue Zhu and Yiwen Zhao for their help during my research. They have provided valuable input in terms of training and/or comments during lab meetings and discussions.

I am grateful to my parents, Mrs. Rupam Kakar and Late Dr. Ashok Kakar for providing me so much support and encouragement to excel in my academic pursuits. They have always been there for me and have provided me all the resources to follow my dreams. A special thanks to my sister, Dr. Smita Kakar and my brother in law, Dr. Vijay Walia for all their help and support throughout these years and even in the past. They have been my inspiration to pursue higher academic honors. I could not have done this without them. 
I would like to thank my friend and lovely wife Nupur. She has been so supportive in this journey from the start. She is very understanding and very caring. I cannot thank her enough.

Last but not the least, I would like to thank all my friends, especially Prathamesh, Alok, Hasan, Priya, Nishan, Adam, Anitha, Kaveendi, Keerthi and Isha who were there besides me in good and bad times to encourage me and motivate me. They reminded me that it is equally important to take a break once in a while. 


\section{DEDICATION}

Dedicated to my hero. One who always believed in me. I miss you.

\section{DAD}




\section{PREFACE}

This dissertation has been written in the manuscript format according to the formatting guidelines of the target journal. It has been divided into three manuscripts that relate to reservoir patch-based powder drug delivery, full-surface powder vaccine delivery, and hydrogel-based transdermal drug delivery. The first manuscript examines AFLassisted high-dose powder drug delivery. This manuscript has been prepared following the Journal of Controlled Release guidelines. The second manuscript is focused on AFL-assisted full-surface powder vaccination in the presence of MPL adjuvant in both newborn and adult mice. This manuscript has been prepared following the Journal of Controlled Release guidelines. The final manuscript explores AFL-assisted hydrogelbased transdermal drug delivery. This manuscript has been prepared following the International Journal of Pharmaceutics guidelines. 


\section{TABLE OF CONTENTS}

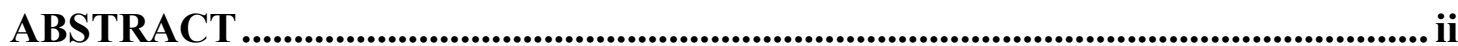

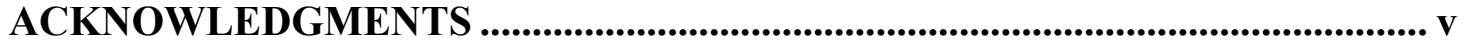

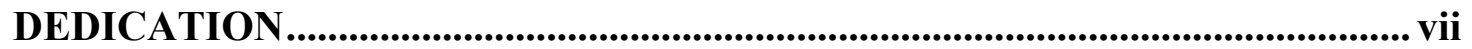

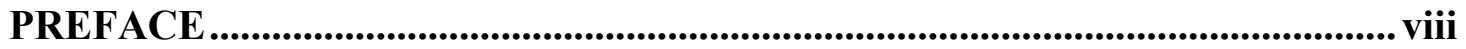

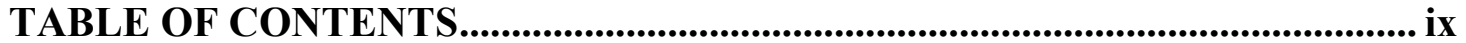

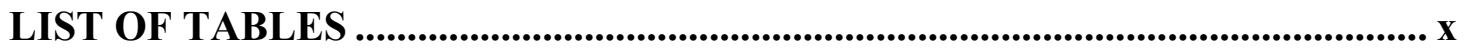

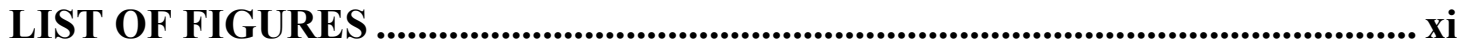

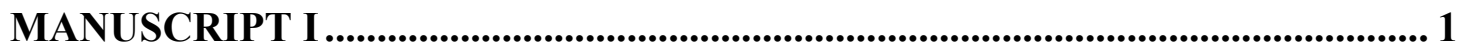

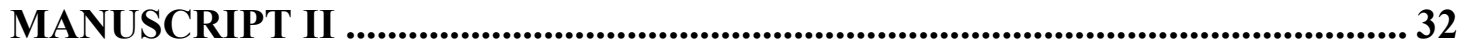

MANUSCRIPT III ...........................................................................................67 67 


\section{LIST OF TABLES}

TABLE

PAGE

Table 1. Tapped coating capacity of different AZT/Mannitol ratios. ....................... 15 


\section{LIST OF FIGURES}

FIGURE

PAGE

\section{MANUSCRIPT I}

Figure 1. Efficient reservoir patch-based powder SRB/mannitol delivery via lasergenerated skin MCs. 13

Figure 2. Delivery kinetics of pure AZT powder in mice.

Figure 3. Efficient reservoir patch-coated powder AZT/mannitol delivery via laser generated skin MCs

Figure 4. Efficient reservoir patch-coated powder AZT/mannitol delivery via laser generated skin MCs.

Figure 5. Efficient reservoir patch-coated powder SRB/mannitol delivery via MN-

generated skin MCs.

Figure 6. Histological analysis of laser and MN-generated skin MCs 21

Figure 7. Sustained bulk delivery of pure OVA 22

\section{MANUSCRIPT II}

Figure 1. Efficient full-surface powder SRB delivery via laser-generated skin MCs. 46

Figure 2. Imaging of SRB full-surface patch in vivo........................................ 47

Figure 3. TEWL and correlation with powder SRB delivery efficiency .................. 48

Figure 4. Efficient full-surface powder OVA delivery via laser-generated skin MCs 49

Figure 5. Size of NP(SRB) measured by Zetasizer Nano ZS90 (Malvern) ............... 50

Figure 6. Efficient full-surface delivery of powder PLGA NPs ............................. 50 
Figure 7. Size of NP(MPL) measured by Zetasizer Nano ZS90 (Malvern).

Figure 8. Comparison of laser-based powder OVA delivery with subcutaneous (SC)

delivery in the presence and absence of NP(MPL)

Figure 9. Minimal local reactions following LPD of OVA in the presence or absence

of NP(MPL) 53

Figure 10. Comparison of laser-based powder Hib vaccine delivery with SC delivery in the presence or absence of NP(MPL) 55

Figure 11. TEWL of laser-treated guinea pig skin. 56

Figure 12. Efficient delivery of powder OVA in guinea pigs 57

\section{MANUSCRIPT III}

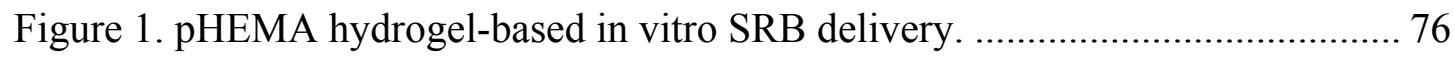

Figure 2. pHEMA hydrogel-based in vivo SRB delivery ......................................... 77

Figure 3. SRB-loaded PVA hydrogel .................................................................... 77

Figure 4. PVA hydrogel-based in vitro SRB delivery …………............................. 78

Figure 5. PVA hydrogel-based in vivo SRB delivery .............................................. 79

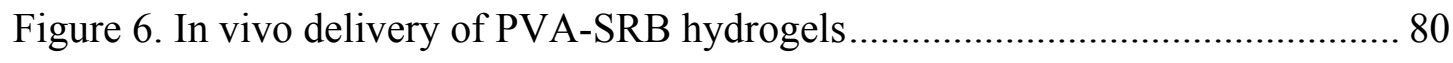

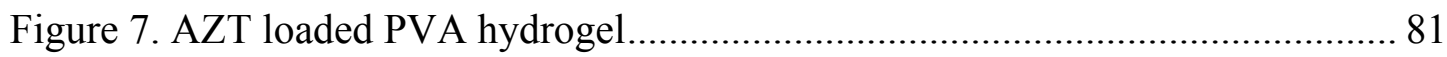

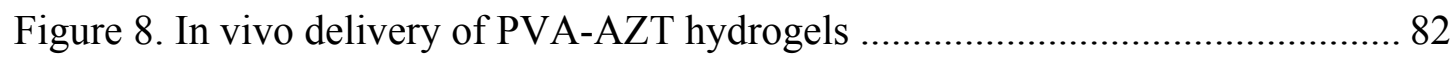




\title{
MANUSCRIPT I
}

\author{
Laser-assisted high-dose powder drug delivery \\ Prateek Kakar, Yan Cao, Yiwen Zhao and Xinyuan Chen \\ (Prepared for submission to Journal of Controlled Release)
}




\section{Laser-assisted high-dose powder drug delivery \\ Prateek Kakar, Yan Cao, Yiwen Zhao and Xinyuan Chen ${ }^{*}$}

Biomedical \& Pharmaceutical Sciences, College of Pharmacy, University of Rhode Island, Kingston, RI

*Address Correspondence to: Xinyuan Chen, Biomedical \& Pharmaceutical Sciences, College of Pharmacy, University of Rhode Island, Kingston, RI 02881,TEL: 401-8745033; FAX: 401-874-5787; xchen14@uri.edu

The authors have no conflict of interest to declare. 


\begin{abstract}
Transdermal delivery is highly attractive for alternative drug delivery due to its noninvasiveness, self-administration, enhanced bioavailability and extended drug release. Current transdermal drug delivery is only limited to low-dose, small, hydrophobic drugs. In this study, we explored ablative fractional laser (AFL)-based powder reservoir patch for high-dose multi-day sustained delivery of hydrophilic drugs in murine models. This novel delivery platform is based on coating powder drugs into reservoir patches followed by topical application onto AFL-generated skin microchannels (MCs) to deliver drug into the skin. Using antiretroviral drug zidovudine (AZT) as a model hydrophilic drug, we found $11.42 \mathrm{mg}$ AZT could be coated per $0.5 \mathrm{~cm}^{2}$ patch area with tapped coating to elicit 3-day sustained release, while $\sim 68 \mathrm{mg}$ AZT could be coated per $0.5 \mathrm{~cm}^{2}$ patch area with compression coating to elicit 6-day sustained release. This study also explored microneedle (MN)-assisted powder reservoir patch delivery and we found AFL was more promising than $\mathrm{MN}$ to support more than 3-day sustained powder drug delivery. This can be attributed to the fact that $\mathrm{AFL}$ is more prone to generate relatively large skin MCs that remain open beyond 3 days. Besides small chemicals, AFL-based powder reservoir patch delivery could also be used for efficient delivery of macromolecules.
\end{abstract}

Keywords: reservoir patch; transdermal drug delivery; ablative fractional laser; powder drug delivery; sustained release; microneedle. 


\section{Graphical abstract}

High-dose reservoir patches efficiently deliver powder drugs via ablative fractional laser-generated skin microchannels (MCs) over 6 days owing to water evaporation from skin MCs to slowly dissolve bulk drug powder.
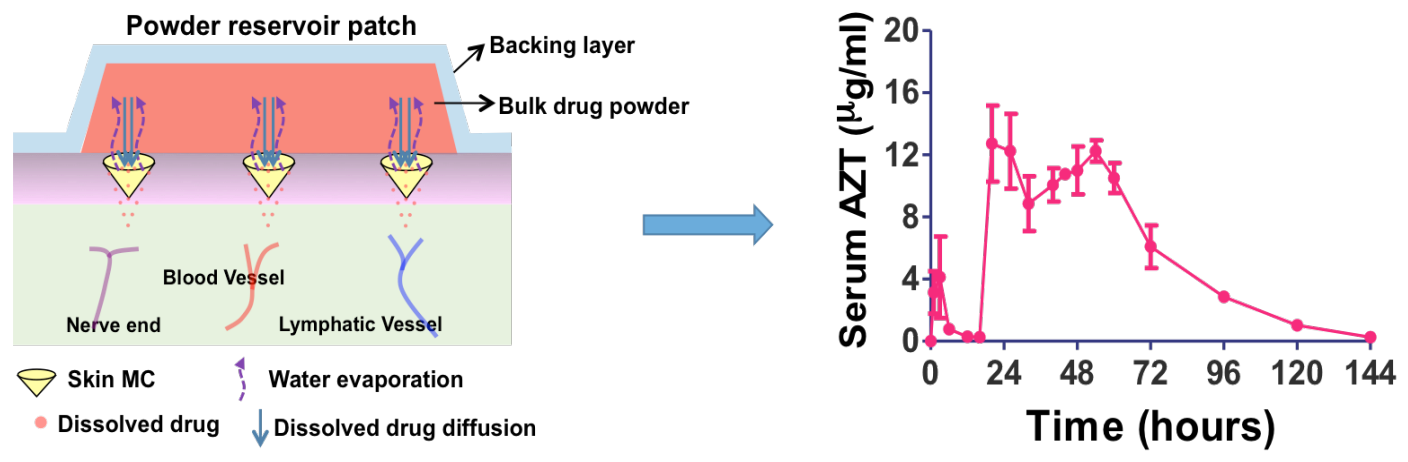


\section{Introduction}

Conventional oral dosage forms, like tablets and capsules, have been the first choice of drug delivery for decades due to the convenience of administration. However, most drugs administered by oral route have poor bioavailability due to hepatic first-pass effect and harsh conditions of the gastrointestinal tract [1].

Transdermal drug delivery is attractive due to the plethora of advantages it offers. It bypasses the hepatic metabolism, increases bioavailability, and can be self-applied. It might also lower drug dose and reduce dosing frequency. Yet only a few hydrophobic drugs can be delivered via transdermal route due to the barrier function of the stratum corneum (SC) layer [2-4]. SC layer is composed of specialized lipid structures that hinder the transmigration of large hydrophobic molecules and almost all hydrophilic molecules. The desirable attributes of drugs for transdermal drug delivery include a) molecular weight below 500 Daltons, b) specific log P coefficient, c) good oil and water solubility [5]. Different methods (e.g., chemical enhancers, iontophoresis, and ultrasound) have been explored to disrupt SC layer to facilitate transdermal drug delivery. Chemical enhancers modify lipid structures of the SC layer to increase drug

permeation and yet can cause skin irritation due to the difficulty to limit their effects to the SC layer [2]. Iontophoresis uses electric fields to mobilize charged molecules across the SC layer and can conveniently control drug release rate by modulation of electrical current [2]. Iontophoresis is most effective to deliver small charged drugs. Ultrasound generates oscillating pressure waves at high frequencies $(\mathrm{MHz})$ or cavitation bubbles at low frequencies $(\mathrm{kHz})$ to disrupt $\mathrm{SC}$ layer to facilitate transdermal drug delivery [6]. Interestingly, low-frequency sonophoresis (LFS) has 
been found to be 3 orders of magnitude more effective than high-frequency sonophoresis (HFS) to increase transdermal drug delivery [6]. The safety and efficacy of LFS to enhance transdermal drug delivery remain to be explored in clinical settings. Lasers have also been explored to facilitate transdermal drug delivery. In early studies, laser beams of a few millimeters in diameter were used to ablate SC layer to facilitate transdermal drug delivery. The very first laser used for drug delivery was a pulsed argon fluoride excimer laser for SC ablation in 1987 [7]. Despite an effective strategy, full-surface laser ablation to facilitate transdermal drug delivery faces safety concerns due to delayed skin recovery. Recently, ablative fractional lasers (AFLs) have been fabricated for improved skin resurfacing with significantly shortened downturn time as compared to full-surface laser ablation. Due to complete ablation of SC layer, skin MCs have been found to greatly facilitate transdermal delivery of small chemicals, macromolecules, and nanoparticles with little restrictions on chemical structure, molecular weight, and particle size [8-12]. Due to the microscale size, skin MCs can be quickly and completely recovered in days [8-12]. The most widely used lasers for skin microporation include erbium-doped yttrium aluminium garnet (Er:YAG) laser, a portable Precise Laser Epidermal System (P.L.E.A.S.E), and carbon dioxide laser $[13,14]$. AFLs have been employed to study drug permeation that includes imiquimod, dextrans, 5-aminolevulinic acid [15-17].

Recently, our group explored AFL-assisted powder drug delivery. Direct delivery of powders eliminates the need of reconstitution and may improve drug stability. In previous studies, powder drugs were coated in the same pattern as skin MCs on either patch surface or volumetric channels of thick patches. AFL-assisted powder drug 
delivery utilizes water absorption from skin MCs as a key component in powder drug delivery. It ablates a few hundred micrometers of skin layer (stratum corneum), which leads to trans-epidermal water loss. This water travels through the powder layers and dissolves it. Dissolved drugs then slowly diffuse into the skin via skin MCs. Surface coating was found to elicit quick drug delivery ( $\sim \mathrm{hr})$, while volumetric coating was found to elicit $\sim 12$ hour sustained drug release, leading to increased drug bioavailability in vivo $[18,19]$. Despite these advantages, current delivery platforms have relatively low coating and delivery capacity, comparable to traditional patches (a few milligrams a day) $[20,21]$. The majority of high-dose drugs cannot be delivered with these technologies.

We explored high-dose powder drug delivery via AFL-generated skin MCs by coating powder drugs into reservoir patches. In this study, we report two types of reservoir patches to induce multi-day sustained drug release. The first type is based on tapped coating $\left(40 \mathrm{mg} / 0.5 \mathrm{~cm}^{2}\right)$ to induce 3-day sustained drug release and the second type is based on compression coating $\left(250 \mathrm{mg} / 0.5 \mathrm{~cm}^{2}\right)$ to induce 6-day sustained drug release. 


\section{Materials and Methods}

\section{Reagents}

Sulforhodamine B (SRB, 230161), mannitol (M4125), ovalbumin (OVA, A5503), texas red ovalbumin (TR-OVA) and zidovudine (AZT, A2169) were purchased from Sigma (St. Louis, MO). 3'-Azido-3'-deoxythymidine (AZT-IS, MG103), AZT internal standard, was purchased from Moravek Biochemicals (Brea, CA).

\section{Animals}

BALB/c mice (female, 6-8 weeks old) were purchased from Charles River Laboratories (Wilmington, MA). They were housed and maintained in the animal quarters of University of Rhode Island. For the experiments, the animals were anaesthetized for hair removal, laser and microneedle treatment, patch application and patch removal. Institutional Animal Care and Use Committees of URI approved all procedures.

\section{Generation of skin MCs}

An UltraPulse Fractional $\mathrm{CO}_{2}$ Laser from Lumenis Inc. (San Jose, CA) was used to generate skin MCs at laser energy (2.5, 5 and 10mJ) and skin coverage (5, 10, 20\%). Stainless steel MNs with $56 \mathrm{MNs}$ in $\sim 1 \mathrm{~cm}$ circular area was kindly provided by Dr. Mark Prausnitz at Georgia Institute of Technology. Each MN was about $700 \mu \mathrm{m}$ in height, $200 \mu \mathrm{m}$ in width, and $50 \mu \mathrm{m}$ in thickness. MNs were pressed into mouse skin, waited for 10 seconds, and then retracted. MN application was repeated for multiple times and each time MN was turned clockwise for 5-10 degrees to avoid insertion into the same MCs. 


\section{Patch preparation, coating and extraction}

For tapped coating, drug powder was loaded into plastic cylindrical container with $8 \mathrm{~mm}$ in diameter and $5 \mathrm{~mm}$ in height and gently tapped. For compression coating, a centrifuge tube was filled with polydimethylsiloxane (PDMS) solution up to $70 \%$ and allowed to dry. A plastic reservoir patch was applied to the top layer of dries PDMS using an adhesive tape. Bulk drug powder was loaded over the plastic patch and was centrifuged at 20,000 rpm for 90 minutes.

\section{Patch application}

Hairless dorsal mouse skin was exposed to laser or MN treatment followed by topical application of the patches, which were then covered with a thin $3 \mathrm{M}$ Tegaderm. A bandage was applied to ensure that the patches stay in position till removal.

\section{Oral gavage}

Oral gavage of AZT was performed following a published protocol [22]. The mice were held vertically and a sterile plastic feeding tube was inserted and advanced into the stomach. The drug solution was slowly injected followed by careful removal of the tube from the stomach.

\section{LC-MS/MS quantification of AZT}

Liquid chromatography-tandem mass spectrometry (LC-MS/MS) was employed to quantify blood AZT levels [23]. Patch extracts and AZT standards $(6.25,12.5,25,50$, 100, 200, $400 \mathrm{ng} / \mathrm{ml}$ ) were mixed with $100 \mathrm{ng} / \mathrm{ml} \mathrm{AZT-Internal} \mathrm{standard} \mathrm{(IS)} \mathrm{Serum}$ samples were diluted 20 times, mixed with $100 \mathrm{ng} / \mathrm{ml}$ AZT-IS and filtered through 10kDa cutoff Amicon filter purchased from EMD Millipore (Burlington, MA) Samples were loaded into an AB Sciex 4500 QTRAP LC-MS/MS equipped with 
Shimadzu LC-20AD pumps and a QTRAP 4500 System. A Synergi Hydro-RP 80A, $2.0 \times 150 \mathrm{~mm}, 4 \mu \mathrm{m}$ particle size analytical column (Phenomonex, Torrance, $\mathrm{CA}$ ) was used for sample separation. Acquisition was performed in multiple reactionmonitoring (MRM) mode using m/z 268/127 for AZT and 271/130 for AZT-IS detection. A standard curve was generated by plotting peak area ratios of AZT to AZT-IS against AZT concentrations and used to quantify AZT levels in the unknown samples.

\section{Statistical analysis}

All values were expressed as Mean $\pm \operatorname{SEM}$ (standard error of mean). Student t-test was used to analyze the difference between mice groups. P value was calculated using PRISM software (GraphPad, San Diego, CA) and considered significant if it was less than 0.05 . 


\section{Results}

\section{Reservoir patch-based powder SRB delivery with tapped coating}

Considering some drugs have relatively high doses (tens to hundreds of milligrams), 'bulk' drug powder was coated into reservoir patches for delivery via laser-generated skin MCs. Readily detectable SRB was used in this study. SRB has been reported to have a $50 \%$ lethal dose (LD50) of $887 \mathrm{mg} / \mathrm{kg}$ in mice (i.e., $22 \mathrm{mg}$ SRB given to $25 \mathrm{~g}$ mice will cause $50 \%$ death) [24]. To avoid potential toxicity, SRB was mixed with mannitol at 1:20 weight ratio and then lyophilized for reservoir patch coating and delivery. We found $\sim 1.9 \mathrm{mg}$ SRB and $\sim 38 \mathrm{mg}$ mannitol could be coated per reservoir patch and this SRB dose was not expected to pose any safety risks in mice. Powder $\mathrm{SRB} /$ mannitol-coated reservoir patches were topically applied onto laser-treated skin. Trans-epidermal water loss (TEWL) plays an important role in the delivery of powder drugs via laser-ablated skin MCs. We carried out drug delivery at $2.5 \mathrm{~mJ}$ laser energy and different percentage coverages $(5,10,20 \%)$ that vary in TEWL. As shown in Figure 1A, reservoir patches induced highly efficient SRB delivery and the delivery lasted for 1-3 days, depending on laser conditions. Delivery efficiency reached more than $90 \%$ within 24 hours in $20 \%$ laser coverage group, within 48 hours in $10 \%$ laser coverage group, and within 72 hours in 5\% laser coverage group (Figure 1A), indicating a positive correlation between reservoir patch-based powder delivery and TEWL. This study supports modification of laser conditions to modulate duration of powder drug delivery via AFL-generated skin MCs.

Skin images were taken right after patch removal at day 3 . There were no local reactions post patch removal (Figure 1B). 
Besides efficient delivery, quick resealing of skin MCs at the end of delivery can minimize potential environmental pathogen infections. To explore the temporal MC resealing, we measured TEWL at different times after patch removal in 5\% laser coverage group. As shown in Figure 1C, TEWL reduced from $\sim 85 \mathrm{~g} / \mathrm{hm}^{2}$ at the beginning of delivery to $\sim 40 \mathrm{~g} / \mathrm{hm}^{2}$ at the end of the 3-day delivery, hinting partial resealing of skin MCs during the 3-day period. After patch removal, TEWL reduced to baseline levels $\left(\sim 6 \mathrm{~g} / \mathrm{hm}^{2}\right)$ within 36 hours (Figure 1C), indicating complete resealing of skin MCs within this period.

Reservoir patch images were also taken to explore kinetics of powder dissolution and delivery in 5\% laser coverage group. SRB and mannitol were mixed at 1:50,000weight ratio for easy differentiation of dissolved from dry forms of SRB. As shown in Figure 1D, powder SRB/mannitol showed mainly mannitol color at the beginning of the delivery due to its dominant content and then pink color at 4 hours due to the absorption of evaporated water by intermixed SRB powder. With continuous water absorption and increase of moisture levels, SRB/mannitol powder changed to a wet mass at 12 hours (Figure 1D). The wet mass gradually reduced in volume and became complete liquid at 60 hours. Significant reduction of pink color of the liquid from 60 to 72 hours hinted complete delivery of SRB at 72 hours (Figure 1D). 
A

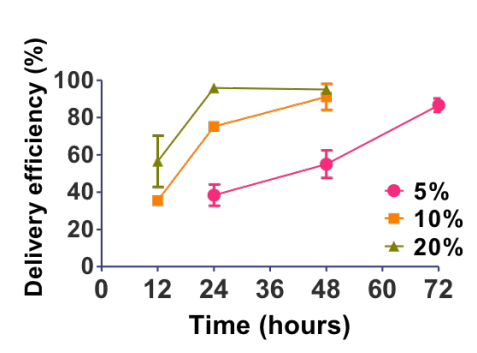

B

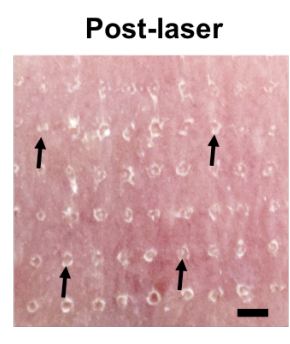

C
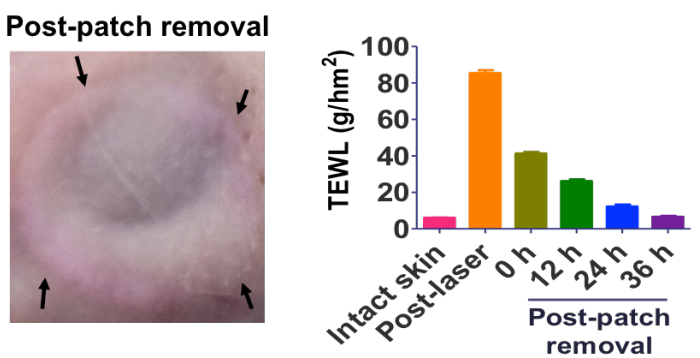

D
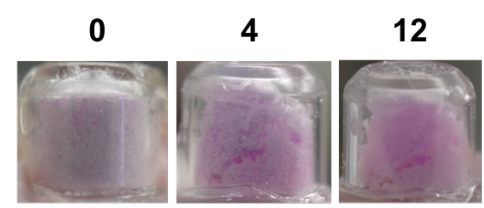

24

36
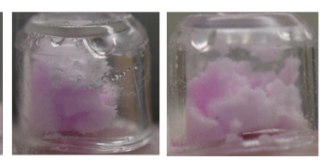

48

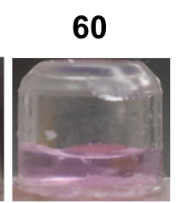

$72 \mathrm{hr}$
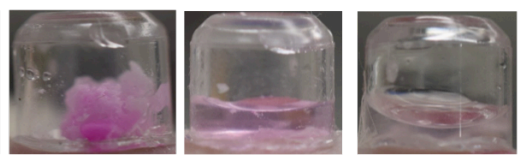

Figure 1. Efficient reservoir patch-based powder SRB/mannitol delivery via lasergenerated skin MCs

A. Lateral back skin of BALB/c mice was exposed to $2.5 \mathrm{~mJ}$ laser treatment at $5 \%$, $10 \%$ or $20 \%$ coverage followed by topical application of powder SRB/mannitol-coated reservoir patches. Patches were removed at indicated times and delivery efficiency was then quantified. $n=2-5$. B. Skin images right after laser treatment of $2.5 \mathrm{~mJ} / 5 \%$ and post-patch removal at day 3. C. TEWL was measured before (Nä̈ve skin) or right after laser (2.5mJ 5\%) treatment, or at different times after patch removal on day 3. n=3. D. Powder SRB/mannitol-coated reservoir patches were topically applied onto laser (2.5mJ /5\%)-treated skin. Patch pictures were taken at different times. Experiments were repeated 3 times and representative patch pictures were shown.

\section{Reservoir patch-based AZT delivery with tapped coating}

We found coating pure powder AZT into reservoir patches only achieved $4.6 \%$ delivery efficiency at the end of 2-day delivery (Figure 2). Thus, small sugar excipient mannitol was mixed with AZT at different ratios to explore whether it could enhance the delivery efficiency. AZT/mannitol in different ratios were coated in reservoir 
patches with tapped coating to calculate average loading $(n=6)$ as shown in table 1 . These patches contained $20 \mathrm{mg}, 11.62 \mathrm{mg}$ and $6.66 \mathrm{mg}$ in 1:1, 1:2.5 and 1:5 mixing ratios, respectively. Around $40 \mathrm{mg}$ AZT/mannitol (Table 1) was found to be coated per reservoir patch with tapped coating. Laser energy of $5 \mathrm{~mJ}$ and $5 \%$ skin coverage was used for all patch deliveries. An $8 \times 8 \mathrm{~mm}^{2}$ area of female BALB/c mouse skin was exposed to laser treatment and the patch was then topically applied and secured in place. Serum AZT concentration was quantified using LC/MS/MS. Serum AZT levels peaked at around 16-20 hours and then slowly reduced to baseline levels in 3 days (Figure 3A-3C). Oral gavage delivery of $0.32 \mathrm{mg}$ AZT was used for comparison. Serum AZT levels peaked at 30 minutes and declined to baseline within 3 hours of oral delivery (Figure 3D). The remaining AZT in laser-based patch delivery was quantified and compared with total coating amount to determine the delivery efficiency. We found approximately $51 \%, 93 \%$ and $90 \%$ delivery efficiency could be obtained at 1:1, 1:2.5 and 1:5 ratios, respectively (Figure 3E). A typical powder AZT/mannitol coated reservoir patch is shown in Figure 3F.

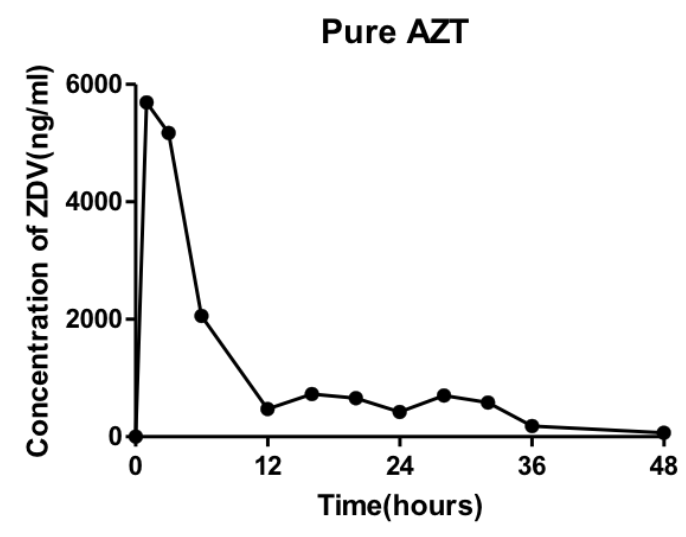

Figure 2. Delivery kinetics of pure AZT powder in mice at $5 \mathrm{~mJ} / 5 \%$ laser condition. 
Table 1. Tapped coating capacity at different AZT/Mannitol ratios.

\begin{tabular}{|c|c|c|}
\hline $\begin{array}{c}\text { Ratio of AZT/Mannitol } \\
\text { (weight:weight) }\end{array}$ & $\begin{array}{c}\text { Tapped coating capacity } \\
\text { (Mean } \pm \\
\text { Standard deviation) (mg) }\end{array}$ & $\begin{array}{c}\text { AZT amount } \\
\text { (mg) }\end{array}$ \\
\hline $1: 1$ & $40.68 \pm 3.29$ & 20 \\
\hline $1: 2.5$ & $39.75 \pm 3.55$ & 6.66 \\
\hline $1: 5$ & $39.41 \pm 2.59$ & \\
\hline
\end{tabular}



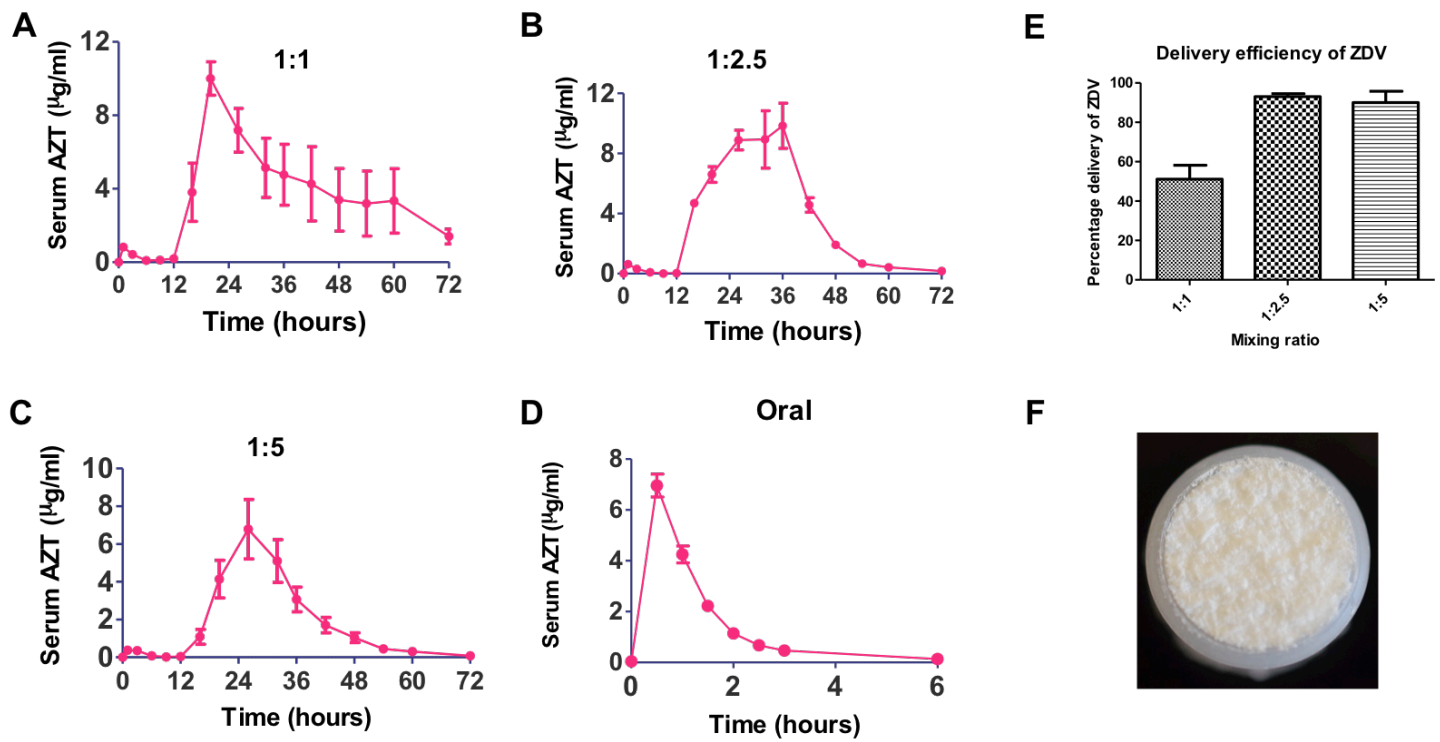

Figure 3. Efficient reservoir patch-coated powder AZT/mannitol delivery via laser generated skin MCs

A-C. Lateral back skin of BALB/c mice was exposed to $5 \mathrm{~mJ}$ laser treatment at $5 \%$ followed by topical application of powder AZT/mannitol-coated reservoir patches at 1:1 (A), 1:2.5 (B), and 1:5 ratios (C). Patches were removed at day 3 and delivery efficiency was then quantified. $n=3 \mathbf{D}$. Oral gavage delivery of AZT solution in mice. $n=3 \boldsymbol{E}$. Delivery efficiency of the three ratios. $\boldsymbol{F}$. Close-view of AZT/mannitol-coated reservoir patch using tapped coating method at 1:2.5 mixing ratio.

\section{Reservoir patch-based powder AZT delivery with compression coating}

We next explored whether powder reservoir patches with compression coating could achieve high-efficient delivery and more extended drug release. Lateral back skin of mice was exposed to laser treatment (10 $\mathrm{mJ}$ and 5\% skin coverage) followed by topical application of the reservoir patches with compression coating at 1:2.5 AZT/mannitol ratio. Serum AZT levels were quantified as above. Similar patterns of delivery were observed except duration of delivery was extended to 6 days (Figure 
4A). We also measured delivery efficiency by quantification and comparison of patchcoated and remained AZT on day 6 . We found approximately $94 \%$ AZT was delivered in 6 days. A typical powder reservoir patch following compression coating is shown in Figure 4B.

A

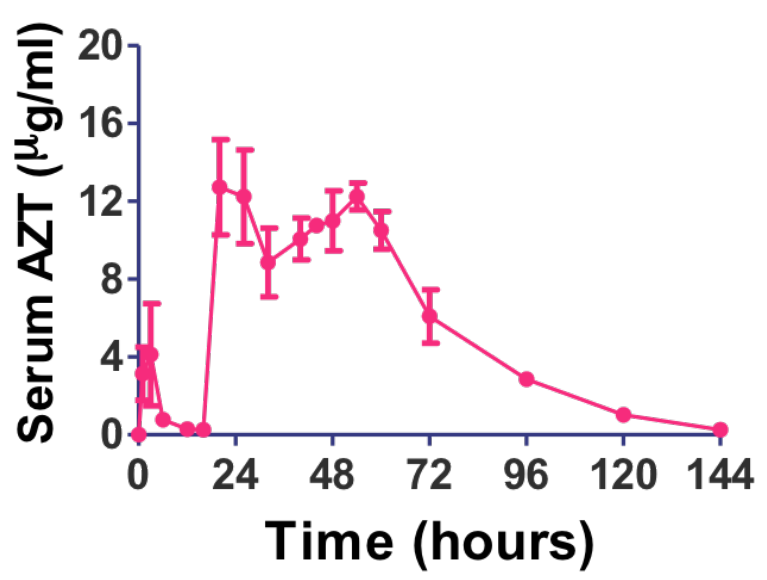

B

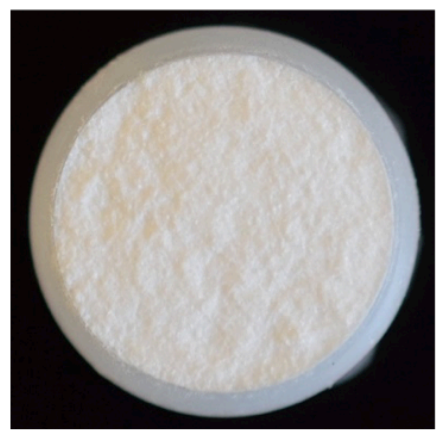

Figure 4. Efficient reservoir patch-coated powder AZT/mannitol delivery via laser generated skin MCs

A. Lateral back skin of BALB/c mice was exposed to $10 \mathrm{~mJ}$ laser treatment at $5 \%$ coverage followed by topical application of powder AZT/mannitol-coated reservoir patches (compression coating, 1:2.5 ratio). Patches were removed at day 6 and delivery efficiency was then quantified. $n=3 \boldsymbol{B}$. Close-view of AZT/mannitol-coated reservoir patch using compression coating method. 


\section{Powder reservoir patch delivery via MN-generated skin MCs}

Besides laser, MNs use microscale needles to puncture the skin to create skin MCs, which have been also explored to facilitate transdermal drug delivery [10]. The ability of MN-generated skin MCs to facilitate reservoir patch-based powder delivery was also explored here. Stainless steel MNs as shown in Figure 5A were used to generate skin MCs. Due to crucial roles of water evaporation in transdermal powder delivery based on our previous experience, we first explored whether MN treatment could significantly increase TEWL value of the skin. We found quick retraction of MNs after insertion only slightly increased TEWL, while allowing MNs to stay in the skin for 10 seconds could significantly increase TEWL (data not shown). We found $10 \mathrm{MN}$ applications could generate a similar TEWL to that generated by laser $(2.5 \mathrm{~mJ} 5 \%)$, while 3 and $6 \mathrm{MN}$ applications generated lower TEWL values (Figure 5B). Powder SRB-coated reservoir patches (tapped coating) were topically applied onto the different MN-generated skin MCs. As shown in figure 5C, $10 \mathrm{MN}$ applications achieved more than $85 \%$ delivery efficiency at 72 hours, while 3 and $6 \mathrm{MN}$ applications induced less than $25 \%$ delivery efficiency within the same period. Skin images were taken right after $10 \mathrm{MN}$ treatments and right after patch removal on day 3. MN insertion sites were clearly visible, however MN-generated skin MCs were not visible by naked eye right after treatment (left, Figure 5D). Right after patch removal, signs of circular patch edges on skin were clearly visible and no other visible local reactions could be seen except slightly white-colored skin in patch-applied area (right, Figure 5D). 
A

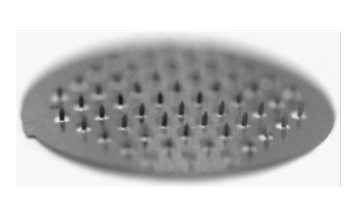

B

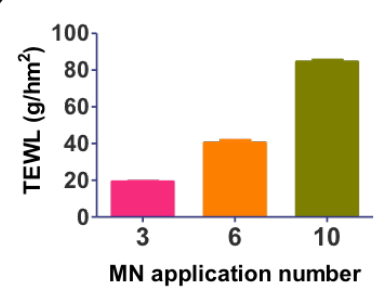

D

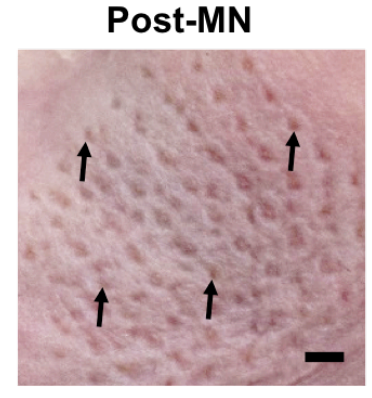

Post-patch removal

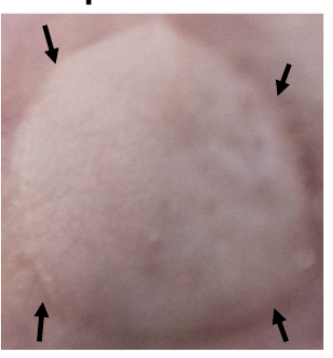

C ฐ

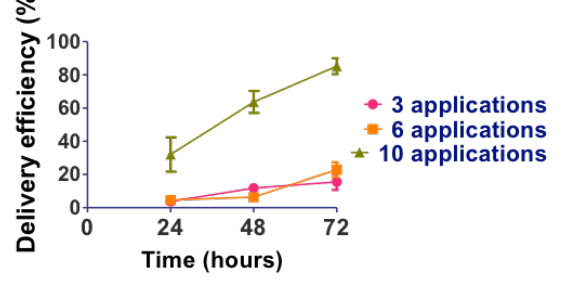

E

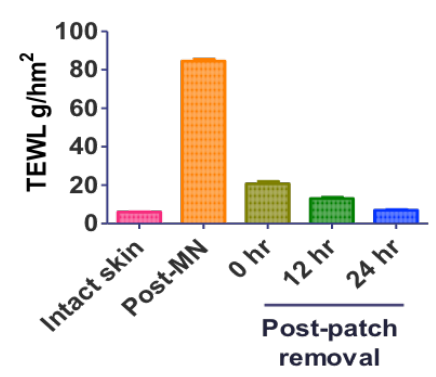

Figure 5. Efficient reservoir patch-coated powder SRB/mannitol delivery via MNgenerated skin MCs

A. Pictures of stainless steel MNs used in this study. Each device contains 56 MNs in 1-cm diameter circular area. B. TEWL of MN-treated skin after 3, 6, and 10 applications. MNs were allowed to remain in the skin for 10 seconds before retraction. C. Powder SRB/mannitol-coated reservoir patches were topically applied on MN-treated skin. Patches were removed at different times and delivery efficiency was then quantified. $n=3$. D. Skin images right after $10 M N$ treatments and right after patch removal at day 3. E. TEWL before or right after $10 \mathrm{MN}$ applications, or at different times after reservoir patch removal at day 3. $n=3$.

The timelines of $\mathrm{MC}$ resealing were also explored. As shown in figure 5E, TEWL significantly reduced from $\sim 84 \mathrm{~g} / \mathrm{hm}^{2}$ at the beginning of delivery to $\sim 20 \mathrm{~g} / \mathrm{hm}^{2}$ at the end of the 3-day delivery in $10 \mathrm{MN}$ applications group, hinting significant resealing of skin MCs in the 3-day period. TEWL then reduced to baseline levels within 24 hours 
after patch removal in $10 \mathrm{MN}$ applications group (Figure 5E). This study indicated that MNs could elicit similar delivery patterns if they induced similar levels of TEWL to laser ablation.

\section{Histological analysis of laser and MN-generated skin MCs}

The above studies indicated that both laser and MNs could be used to generate skin MCs to support efficient powder reservoir patch delivery. In our studies, similar delivery efficiencies (Figure 1A \& 5C) were observed when laser and MNs generated similar TEWL (Figure 1C \& 5E). Considering laser (2.5mJ 5\%) generated $11 \times 11$ array of skin MCs in $8 \times 8 \mathrm{~mm}^{2}$ area, while $10 \mathrm{MN}$ applications were estimated to create 560 skin MCs in a similar skin area assuming each MN application generated completely new skin MCs. The higher skin MC density in MN group hinted MNgenerated skin MCs might be smaller than laser-generated ones. To prove this, we conducted skin histological analysis of laser- and MN-generated skin MCs. Lateral back skin of mice was exposed to laser $(2.5 \mathrm{~mJ} / 5 \%)$ or $\mathrm{MN}$ treatment (10 applications) and skin was dissected right after treatment, fixed in formalin and then subjected to paraffin sectioning and H\&E staining (conducted at RI Hospital, Molecular pathology department). We found we could easily identify laser-generated skin MCs but not MN-generated skin MCs (Data not shown). To accurately compare the relative size of laser- and MN-generated skin MCs, full-surface powder SRB patches were topically applied onto laser- or MN-treated skin. Skin was dissected 60 minutes later and subjected to cryosectioning (RI hospital). With the help of readily detectable SRB, we were able to identify MN-generated skin MCs, whereas laser-generated skin MCs 
were much bigger (Figure 6). This result explained quicker sealing of MN-generated skin MCs in figure $5 \mathrm{E}$.

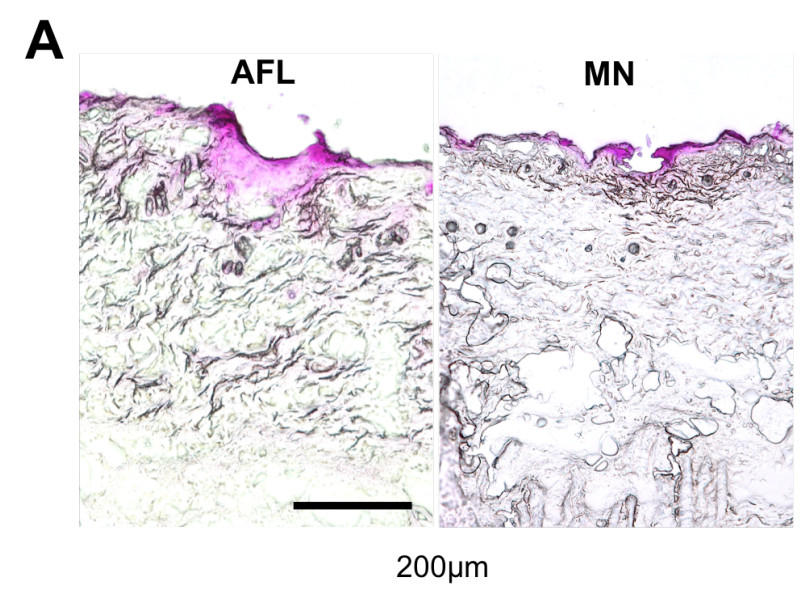

B

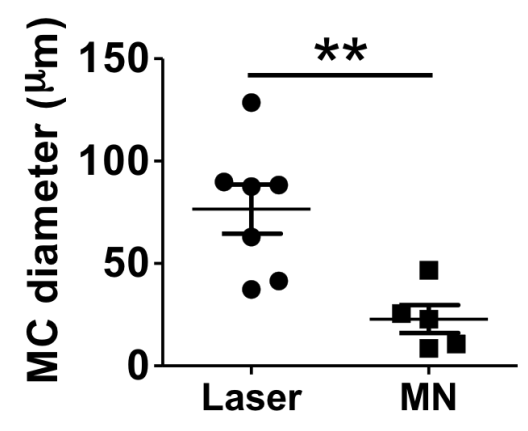

Figure 6. Histological analysis of laser and MN-generated skin MCs

A. Histology images of laser- and MN-treated mouse skin. B. Diameter of microchannels for laser and MN treated skin samples. Laser generated bigger microchannels in the skin compared to $M N$ which explains the longer duration of drug delivery via skin MCs. $n=6$.

\section{Reservoir patch-based powder ovalbumin delivery with tapped coating}

Lastly, the potential of laser-based powder reservoir patch for high-dose macromolecule delivery was also explored. OVA with a molecular weight of $\sim 43 \mathrm{kDa}$ was used as a model. We found that $\sim 20 \mathrm{mg}$ of lyophilized OVA could be coated into the reservoir patches with tapped coating. Powder OVA patches were then topically applied onto laser (2.5mJ/5\%)-treated skin of mice. Patches were removed at different time points and patch-remained OVA was quantified by BCA assay. We found 38, 46 and $70 \%$ of OVA was delivered at 24,48 and 96 hours, respectively as shown in Figure 7. 


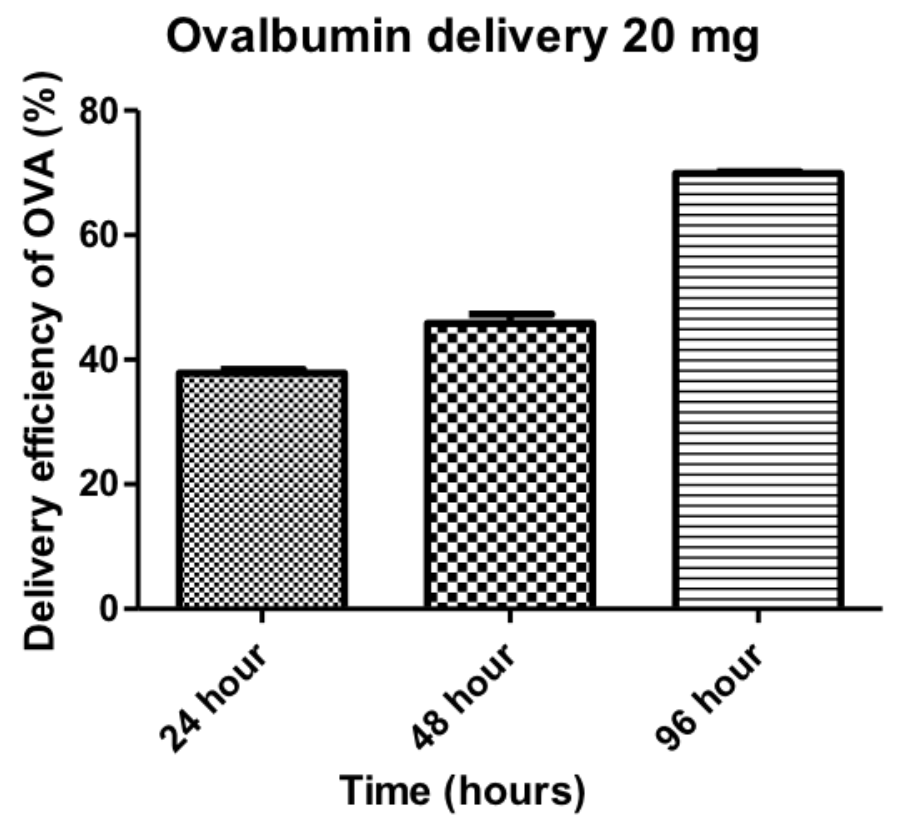

Figure 7. Sustained bulk delivery of pure OVA

$20 \mathrm{mg}$ of lyophilized ovalbumin was loaded in tapped coated reservoir patches and delivered using $2.5 \mathrm{~mJ}$ energy and 5\% coverage. Delivery efficiency was measured at 24, 28 and 96 hours. $n=2$. 


\section{Discussion}

In this study, we explored powder-coated reservoir patches for high-dose drug delivery via AFL-generated skin MCs. Our previous studies explored volumetric coating of AZT to elicit 12-hour sustained release via AFL-generated skin MCs [19]. In this study, we found reservoir patch coating could significantly increase drug coating and delivery capacity and at the same time extend drug release to multiple days. We found $250 \mathrm{mg} \mathrm{AZT/mannitol} \mathrm{powder} \mathrm{could} \mathrm{be} \mathrm{coated} \mathrm{per} \mathrm{reservoir} \mathrm{patch}$ with $0.5 \mathrm{~cm}^{2}$ surface area for efficient delivery via laser-generated skin MCs. It's estimated that $\sim 5 \mathrm{~g}$ drug powder equivalent to approximately 1.4 gram active drug based on current study of AZT can be coated into reservoir patches with $10 \mathrm{~cm}^{2}$ surface area for efficient delivery via laser-generated skin MCs. Such a delivery capacity is expected to significantly expand drugs that can be delivered via transdermal route.

Our current study confirmed importance of water evaporation in laser-assisted powder drug delivery. SRB imaging study (Figure 1D) discovered the dynamics of powder dissolution via laser-generated skin MCs. In more detail, entire powder gradually increased moisture levels, then transformed into a wet mass, and finally transformed into a liquid form. We also observed positive correlation between the duration of delivery and TEWL of the skin in both laser and MN-assisted powder deliveries (Figure 1A and 5C). MN-assisted powder SRB delivery indicated the presence of a threshold TEWL to enable high-efficient powder drug delivery as we found 3 and 6 MN applications induced incomplete SRB delivery (Figure 5C). In the case of incomplete powder delivery, powder would remain in the bottom of the patch, 
indicating the initiation of delivery from powder in close proximity to skin MCs. If TEWL is above the threshold levels, increase of laser energy or percent coverage is expected to increase the rate of drug delivery.

AFL-based powder reservoir patch induced 3-phase drug release. In the first phase, relatively small amount of AZT $(<5 \%)$ was delivered in 12-16 hours after patch application with relatively low serum AZT levels at 1:2.5 AZT:mannitol mixing ratios (Figure 3B and 4A). In the second phase, serum drug levels quickly increased to peak and then maintained at relatively high levels for 12 or 24 hours in case of tapped and compression coating, respectively (Figure 3B and 4A). In the third phase, serum drug levels gradually reduced to baseline levels in the next 1.5 to 3 days (Figure 3B and 4A). Such a delivery pattern follows first-order drug delivery and drug delivery rate was controlled by local drug concentrations. It remains to be explored whether more extended drug release could be elicited by increase of compression forces during patch coating.

Our studies found AZT needed to be mixed with highly water-soluble mannitol to induce high-efficient delivery. AZT has a water solubility of $\sim 25 \mathrm{mg} / \mathrm{ml}$ and mannitol has a water solubility of $216 \mathrm{mg} / \mathrm{ml}$. Due to the slow water evaporation and the 'bulk' powder coating, drugs would have a very high concentration at the early stage of reservoir patch-based powder delivery. Assuming 40mg powder was completely dissolved in the reservoir patch with a calculated volume of $0.25 \mathrm{ml}$, the drug concentration could reach $160 \mathrm{mg} / \mathrm{ml}$. Thus, dissolved drugs were saturated at the beginning of the delivery. The highly saturated drug concentration presents a challenge for continuous drug dissolution and delivery, which justifies the use of 
highly water-soluble small sugar excipients to facilitate powder delivery. In addition, small sugar excipients are expected to have high diffusion efficiency due to their small molecular weight. In our study, we only tested mannitol to support high-efficient laser-based reservoir patch delivery. We believe other small sugar excipients and potentially amino acids can similarly enhance laser-based powder reservoir patch delivery.

Our studies found MNs could also be used to generate skin MCs to support reservoir patch-based powder drug delivery. However, MN-generated skin MCs were much smaller than laser-generated skin MCs (Figure 6B). Thus, MN-generated skin MCs tended to more quickly reseal than laser-generated skin MCs (Figure 1C \& 5E). The quick resealing of $\mathrm{MN}$-generated skin $\mathrm{MCs}$ has been also observed by others and for this reason MNs have been mainly used to generate skin MCs to support short-term drug release $[25,26]$. It remains a challenge to use MNs to generate relatively big skin MCs considering big MNs greatly increase pain sensation [25]. As an alternative strategy, prolonging $\mathrm{MC}$ lifetime has been explored to support more extended (7-day) drug releases $[27,28]$. In our study, we found even the lowest laser condition could generate skin MCs that remained open beyond 3 days. Thus, laser may be a more promising technology to generate skin MCs to support 7-day or even longer sustained drug release.

A small handheld laser device can be manufactured for convenient translation of this technology for high-dose multi-day sustained drug delivery in clinics. Multi-day sustained powder drug delivery is promising to reduce dosing frequency and improve treatment adherence. By limiting skin MCs in the epidermal tissue, laser-based 
reservoir powder patch delivery can also be painless. Powder reservoir patches require no reconstitution and may eliminate cold-chain storage for temperature-sensitive drugs. Powder reservoir patches also have a small package and storage volume. Laserbased powder reservoir patch delivery warrants further investigation for advantageous transdermal drug delivery in humans.

\section{Conclusion}

In summary, we developed a high-dose drug delivery system by coating 'bulk' drug powder into reservoir patches followed by topical application onto AFL-generated skin MCs to initiate transdermal drug delivery. Coating methods can conveniently modulate the coating capacity and duration of delivery. Tapped coating can be employed for 3-day sustained drug delivery, whereas compression coating can be explored for 6-day sustained drug delivery. Laser-based powder reservoir patch delivery induces minimal local reactions and laser-generated skin MCs can be quickly resealed in 1-2 days after completion of the delivery. Our study also supports laser as a more promising technology to generate skin MCs to support more than 3-day sustained drug release. Laser-based powder reservoir patch delivery involves no complex powder formulation and provides a convenient transdermal delivery platform for high-dose multi-day sustained drug delivery. 


\section{Acknowledgements}

This work is partly supported by the National Institutes of Health grants DA033371

and AI107678 (to X.Y.C.). Microplate reader, Nikon Eclipse E600 microscope used in this work are supported by an Institutional Development Award (IDeA) from the National Institute of General Medical Sciences of the National Institutes of Health grant P20GM103430. We thank Ashlee Sturtevant, Molecular pathology department, RI hospital for conduction of paraffin and cryosectioning for us. 


\section{References}

[1] I. Gomez-Orellana, Strategies to improve oral drug bioavailability, Expert opinion on drug delivery, 2 (2005) 419-433.

[2] M.R. Prausnitz, R. Langer, Transdermal drug delivery, Nature biotechnology, 26 (2008) 1261-1268.

[3] A. Naik, Y.N. Kalia, R.H. Guy, Transdermal drug delivery: overcoming the skin's barrier function, Pharmaceutical science \& technology today, 3 (2000) 318-326.

[4] K.S. Paudel, M. Milewski, C.L. Swadley, N.K. Brogden, P. Ghosh, A.L.

Stinchcomb, Challenges and opportunities in dermal/transdermal delivery, Therapeutic delivery, 1 (2010) 109-131.

[5] Prausnitz MR, Mitragotri S, Langer R. Current status and future potential of transdermal drug delivery. Nat Rev Drug Discov. 2004;3:115-124.

[6] S. Mitragotri, Healing sound: the use of ultrasound in drug delivery and other therapeutic applications, Nature reviews. Drug discovery, 4 (2005) 255-260.

[7] Controlled Removal of Human Stratum Corneum by Pulsed Laser (Journal of Investigative Dermatogy, 88(1):88-93, Feb 1987.

[8] M. Haedersdal, F.H. Sakamoto, W.A. Farinelli, A.G. Doukas, J. Tam, R.R. Anderson, Fractional $\mathrm{CO}(2)$ laser-assisted drug delivery, Lasers in surgery and medicine, 42 (2010) 113-122.

[9] J.W. Lee, P. Gadiraju, J.H. Park, M.G. Allen, M.R. Prausnitz, Microsecond thermal ablation of skin for transdermal drug delivery, Journal of controlled release : official journal of the Controlled Release Society, 154 (2011) 58-68. 
[10] G. Levin, A. Gershonowitz, H. Sacks, M. Stern, A. Sherman, S. Rudaev, I. Zivin, M. Phillip, Transdermal delivery of human growth hormone through RFmicrochannels, Pharmaceutical research, 22 (2005) 550-555.

[11] M.R. Prausnitz, Microneedles for transdermal drug delivery, Advanced drug delivery reviews, 56 (2004) 581-587.

[12] A. Kumar, P. Wonganan, M.A. Sandoval, X. Li, S. Zhu, Z. Cui, Microneedlemediated transcutaneous immunization with plasmid DNA coated on cationic PLGA nanoparticles, Journal of controlled release : official journal of the Controlled Release Society, 163 (2012) 230-239.

[13] Scheiblhofer S, Thalhamer J, Weiss R. Laser microporation of the skin: Prospects for painless application of protective and therapeutic vaccines. Expert Opin Drug Delivery 2013; 10(6): 761-773.

[14] Hsiao CY, Sung $\mathrm{HC}$, $\mathrm{Hu} \mathrm{S}$, Huang $\mathrm{CH}$. Fractional $\mathrm{CO}_{2}$ laser treatment to enhance skin permeation of tranexamic acid with minimal skin disruption. Dermatology 2015; 230(3): 269-75.

[15]M. Haedersdal, F.H. Sakamoto, W.A. Farinelli, A.G. Doukas, J. Tam, R.R. Ander son. Fractional CO(2) laser-assisted drug delivery. Lasers Surg. Med., 42 (2010), pp. 113-122.

[16] W.R. Lee, S.C. Shen, S.A. Al Suwayeh, H.H. Yang, C.Y. Yuan, J.Y. Fang. Laserassisted topical drug delivery by using a low-fluence fractional laser: imiquimod and macromolecules, J. Control. Release, 153 (2011), pp. 240-248.

[17] W.R. Lee, S.C. Shen, M.H. Pai, H.H. Yang, C.Y. Yuan, J.Y. Fang. Fractional laser as a tool to enhance the skin permeation of 5-aminolevulinic acid with minimal 
skin disruption: a comparison with conventional erbium:YAG laser, J. Control. Release, 145 (2010), pp. 124-133.

[18] X. Chen, G. Kositratna, C. Zhou, D. Manstein, M.X. Wu, Micro-fractional epidermal powder delivery for improved skin vaccination, Journal of controlled release : official journal of the Controlled Release Society, 192 (2014) 310-316. [19] Y. Cao, P. Kakar, M.N. Hossen, M.X. Wu, X. Chen. Sustained epidermal powder drug delivery via skin microchannels. J. Control. Release, 249 (2017), pp. 94-102. [20 ]Williams A. London: Pharmaceutical Press; 2003. Transdermal and Topical Drug Delivery. [21] Prausnitz MR, Mitragotri S, Langer R. Current status and future potential of transdermal drug delivery. Nat Rev Drug Discov. 2004;3:115-124.

[22] E. Machholz, G. Mulder, C. Ruiz, B.F. Corning, K.R. Pritchett-Corning, Manual restraint and common compound administration routes in mice and rats, J. Vis. Exp. 67 (2012) e2771.

[23] J.E. Rower, B. Klein, L.R. Bushman, P.L. Anderson, Validation of a sensitive LC/MS/MS method for the determination of zidovudine and lamivudine in human plasma, Biomed. Chromatogr. 26 (2012) 12-20.

[24] T.M. Mascari, L.D. Foil, Evaluation of rhodamine B as an orally delivered biomarker for rodents and a feed-through transtadial biomarker for phlebotomine sand flies (Diptera: Psychodidae), Journal of medical entomology, 46 (2009) 1131-1137. [25] J. Gupta, H.S. Gill, S.N. Andrews, M.R. Prausnitz, Kinetics of skin resealing after insertion of microneedles in human subjects, Journal of controlled release : official journal of the Controlled Release Society, 154 (2011) 148-155. 
[26] D.P. Wermeling, S.L. Banks, D.A. Hudson, H.S. Gill, J. Gupta, M.R. Prausnitz, A.L. Stinchcomb, Microneedles permit transdermal delivery of a skin-impermeant medication to humans, Proceedings of the National Academy of Sciences of the United States of America, 105 (2008) 2058-2063.

[27] P. Ghosh, N.K. Brogden, A.L. Stinchcomb, Fluvastatin as a micropore lifetime enhancer for sustained delivery across microneedle-treated skin, Journal of pharmaceutical sciences, 103 (2014) 652-660.

[28] N.K. Brogden, M. Milewski, P. Ghosh, L. Hardi, L.J. Crofford, A.L. Stinchcomb, Diclofenac delays micropore closure following microneedle treatment in human subjects, Journal of controlled release : official journal of the Controlled Release Society, 163 (2012) 220-229. 


\title{
MANUSCRIPT II
}

\author{
Laser-assisted full-surface powder vaccination \\ Prateek Kakar, Yan Cao, Yiwen Zhao and Xinyuan Chen \\ (Prepared for submission to Journal of Controlled Release)
}




\title{
Laser-assisted full-surface powder vaccination
}

\author{
Prateek Kakar, Yan Cao, Yiwen Zhao, and Xinyuan Chen ${ }^{*}$
}

Biomedical \& Pharmaceutical Sciences, College of Pharmacy, University of Rhode Island, Kingston, RI

*Address Correspondence to: Xinyuan Chen, Biomedical \& Pharmaceutical Sciences, College of Pharmacy, University of Rhode Island, Kingston, RI 02881,TEL: 401-8745033; FAX: 401-874-5787; xchen14@uri.edu

The authors have no conflict of interest to declare. 


\begin{abstract}
Needle injections have been the major method of vaccine delivery for a long time. Yet needle-based vaccination has the following drawbacks, like needle-phobia, needlestick injuries, and sharps wastes. Needle-free transdermal delivery is highly attractive for alternative vaccine delivery. Ablative fractional laser (AFL)-based full-surface powder vaccination was explored in this study for needle-free vaccine delivery and adjuvantation. In this platform, powder vaccines were coated onto full-surface adhesive patches followed by topical application onto laser-generated skin microchannels (MCs) to deliver vaccines into the skin. We found full surface-coated powder model antigen ovalbumin (OVA) and haemophilus influenzae type b (Hib) vaccine could be efficiently delivered via AFL-generated skin MCs and elicit comparable immune responses to needle-based subcutaneous delivery. To further boost vaccination, clinical lipophilic monophosphoryl lipid a (MPL) adjuvant was encapsulated into poly(lactic-co-glycolic acid) nanoparticles (NPs) and then coated together with OVA or Hib vaccine on full-surface adhesive patches for efficient delivery via AFL-generated skin MCs. AFL-assisted full-surface powder OVA and haemophilus influenzae type $b$ (Hib) vaccine delivery in the presence of NP(MPL) elicited more than 70-fold higher anti-OVA and anti-Hib antibody titer than needlebased subcutaneous delivery of OVA or Hib vaccine alone. AFL-assisted full-surface powder vaccination showed a good safety with minimal local reactions and quick skin MC resealing (1-2 days). The safe and highly immunogenic AFL-assisted full-surface powder vaccination in the presence of MPL adjuvant warrants further investigation for clinical use.
\end{abstract}


Keywords: transdermal vaccination; transdermal patch; laser; powder delivery; micropore; microchannel; transepidermal water loss. 


\section{Graphical abstract}

Full-surface powder patches efficiently deliver vaccines and monophosphoryl lipid a (MPL) adjuvant via ablative fractional laser-generated skin microchannels (MCs) to induce more potent immune responses than needle-based subcutaneous (SC) injection of vaccine alone.
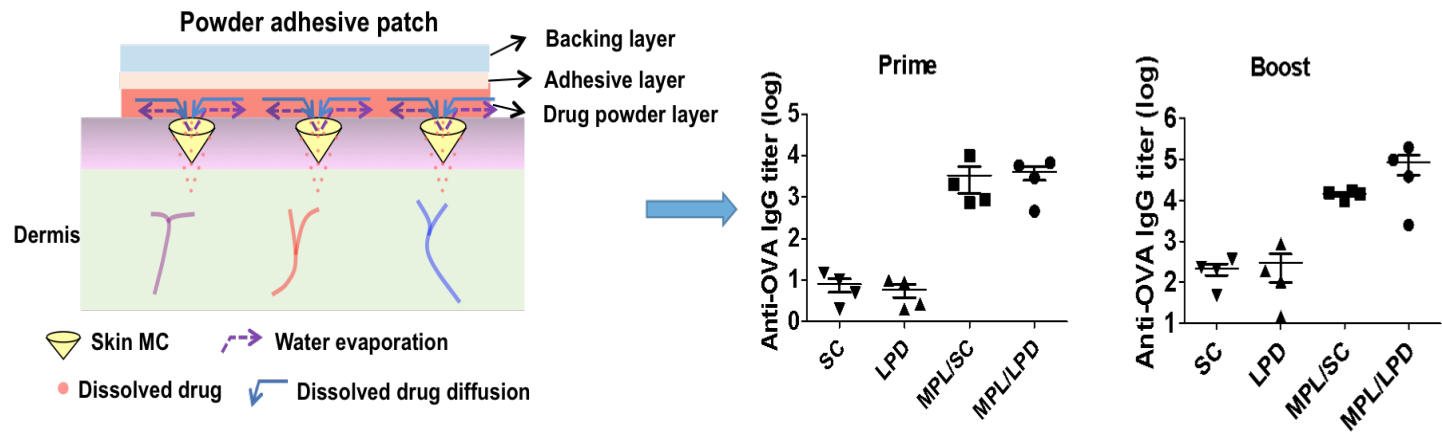


\section{Introduction}

The majority of vaccines are administered by intramuscular needle injections to induce immunological memory to protect against later infections [1]. Needle-based vaccination has the following disadvantages, such as pain, needle-stick injuries, generation of sharp waste and poor patient compliance, especially in children [2,3].

Pediatric patients are more prone to be anxious about getting a needle injection and it can lead to unwanted stress [4]. Intramuscular vaccine delivery often induces relatively weak immune responses due to the rare antigen-presenting cells (APCs) residing in the muscular tissue.

There has been a recent interest in intradermal (ID) or transdermal vaccination due to the presence of a large number of APCs in the skin, such as epidermal Langerhans Cells (LCs) and various types of dermal dendritic cells (DCs) [5-8]. Modification of vaccine administration from IM to ID route by hypodermic needles was found to induce more potent immune responses against influenza vaccine, rabies vaccine, and hepatitis b vaccine [9-11]. Due to the thin skin tissue, hypodermic needle-based ID vaccination faces significant challenges to accurately deliver vaccines into the dermal tissue. To address this, ID adaptors and hollow microneedles (MicronJet600) were developed for use with traditional syringes for accurate ID vaccine delivery [12]. ID microinjection systems as a standalone ID delivery device with prefilled influenza vaccines have been also fabricated to save vaccine dose [13]. Needle-free Jet Injectors have been also developed for IM, subcutaneous (SC), or ID delivery depending on settings [14]. Besides ID delivery technologies, transdermal delivery technologies, like coated and dissolving microneedles (MNs), were also fabricated for vaccine delivery 
$[15,16]$. Dissolving microneedles were fabricated and tested in human volunteers in Phase 1 clinical trials with promising results generating similar or even higher immune responses compared to IM injections [17]. These innovative vaccine delivery technologies use ultra-fine and -short needles or completely eliminate needle use for more patient compliant vaccination. Most of these technologies were found to induce comparable immune responses to needle-based ID or IM delivery.

Ablative fractional laser (AFL) has been also explored to facilitate transdermal vaccine delivery [18]. AFL is fabricated based on the concept of Fractional Photothermolysis, in which a focused array of laser beams generates skin MCs in the range of ten to hundred micrometers in diameter and depth [19]. These MCs have been found to facilitate transdermal delivery of macromolecules, including vaccines. Due to the sparing of majority of the skin from laser ablation, AFL causes minimal skin reactions and induces a fast recovery within $1-2$ days $[18,19]$. In one study, an erbiumdoped yttrium aluminium garnet (Er:YAG) laser was employed to induce systemic antibody production [20]. In another study, a handheld Precise Lasers Epidermal System (P.L.E.A.S.E ${ }^{\circledR}$ ) laser was used to generate skin MCs for allergy vaccine delivery in murine models [21].

Due to the convenience of direct powder delivery, our group previously explored coating powder vaccines in the same pattern as Lumenis Ultrapulse $\mathrm{CO}_{2}$ lasergenerated skin MCs for transdermal vaccination [22]. We found delivery of highly reactive powder BCG vaccines into separated skin MCs induced minimal local reactions as compared to ID injection delivery [22]. Similarly, we found delivery of powder ovalbumin (OVA) in the presence of LPS/CpG adjuvant into AFL-generated 
skin MCs also induced minimal reactions, while needle-based ID injection delivery induced significant local reactions, like erythema, swelling, and ulceration [22]. In these studies, microfractional BCG vaccine and OVA/LPS/CpG delivery via lasergenerated skin MCs were found to induce comparable immune responses to needlebased ID delivery [22].

In this study, we explored AFL to facilitate full-surface-coated powder vaccine delivery to avoid alignment between vaccine coating spots and skin MCs. This exploration was also inspired by our observation of the high potency of water evaporation from skin MCs to dissolve 'bulk' drug powder in reservoir patches in Chapter I. The water evaporation should be also able to dissolve full surface-coated vaccine powder to induce potent immune responses. This study also explored whether clinical monophosphoryl lipid A (MPL) adjuvant could be delivered via AFLgenerated skin MCs to boost vaccination. MPL, a nontoxic derivative of lipopolysaccharide (LPS), is a highly potent Toll-Like Receptor 4 (TLR4) agonist and an important component of combinatorial adjuvants, like AS04 (adsorption of MPL onto Alum adjuvant), or adjuvant systems, like AS01 (MPL and QS21 in liposome), and AS02 (MPL and QS21 in oil-in-water emulsion) [23]. AS04 adjuvant has been approved for incorporation into human papillomavirus (HPV) vaccine (Cervarix) and HBV vaccine (Fendrix) [24,25] and AS01 has been approved to boost malaria RTS,S vaccine efficacy [26]. Due to its relatively high molecular weight (synthetic MPL: $\sim 1763 \mathrm{Da}$ ), transdermal delivery of MPL has been challenging (unpublished data). Due to the aqueous feature of AFL-generated skin MCs, we explored encapsulation of 
MPL into poly(lactic-co-glycolic acid) (PLGA) nanoparticles (NPs) for delivery via AFL-generated skin MCs.

We explored laser-based full-surface powder delivery (LPD) of OVA and haemophilus influenzae type $b$ (Hib) vaccine in the presence or absence of nanoparticle-encapsulated MPL adjuvant in murine models. We found LPD of OVA and Hib vaccine in the presence of MPL adjuvant induced much higher immune responses than LPD or needle-based subcutaneous (SC) delivery of vaccine alone. LPD also induced minimal local reactions and quick skin MC resealing in 1-2 days. The safe and highly immunogenic LPD of vaccine/MPL warrants further exploration for human use. 


\section{Materials and Methods}

\section{Reagents}

Sulforhodamine B (SRB, 230161), ovalbumin (OVA, A5503), Texas Red-conjugated ovalbumin (TR-OVA), and poly(lactic-co-glycolic acid) (PLGA) polymer (719897, Resomer ${ }^{\circledR}$ RG 502 H, acid terminated, 7,000-17,000 Da), MPL (L6895), poly(vinyl alcohol) (PVA, P8136, 30,000-70,000 Da) were purchased from Sigma (St. Louis, MO). Hib vaccine (ActHIB ${ }^{\circledR}$ ) was obtained from Sanofi Pasteur (Swiftwater, PA).

\section{Generation of skin MCs}

An UltraPulse Fractional $\mathrm{CO}_{2}$ Laser (Lumenis Inc. San Jose, CA) was used to generate skin MCs.

\section{Animals}

BALB/c and C57BL/6 mice (female, 6-8 weeks old) and hairless guinea pigs were purchased from Charles River Laboratories (Wilmington, MA). They were housed and maintained in the animal facilities of the University of Rhode Island (URI). Adult mice were anesthetized by intraperitoneal injection of Ketamine $(80 \mathrm{mg} / \mathrm{kg})$ and Xylazine $(10 \mathrm{mg} / \mathrm{kg})$ for hair removal, laser, patch application and transepidermal water loss (TEWL) measurement. Newborn C57BL/6 mice of both genders were selfbred and used for Hib vaccine delivery when reaching one week old. Newborn mice were briefly anesthetized by intraperitoneal injection of Ketamine $(15 \mathrm{mg} / \mathrm{kg})$ for laser treatment and patch application. All animal-related procedures were approved by the Institutional Animal Care and Use Committees of the URI. 


\section{Lyophilized powder preparation}

OVA was mixed with mannitol at 1:25 weight ratio and then lyophilized in a benchtop freeze dry system (FREEZONE, Labconco). To prepare lyophilized OVA/NP(MPL) powder, OVA $(62.5 \mu \mathrm{g})$ was mixed with NP(MPL) $(29.2 \mathrm{mg})$ in water and then lyophilized. To prepare Hib/NP(MPL) lyophilized powder, Hib vaccine (12.5 $\mu \mathrm{g})$ dissolved in accompanying dissolving buffer was mixed with NP(MPL) (29.2mg) and then lyophilized. Due to the presence of salt, sucrose and other excipients in commercial lyophilized Hib vaccine and dissolving buffer, the lyophilized powder weighed $\sim 41.7 \mathrm{mg}$.

\section{Patch powder coating}

A 3M Tegaderm film was layered on top of a scotch packaging tape (3M) to form a sterile adhesive surface for powder coating. Patch adhesive layer was pressed onto 'bulk' SRB or TR-OVA/OVA-mixed powder for an even full-surface coating. Patches were then cut into small pieces $\left(3 \times 3 \mathrm{~mm}^{2}\right)$ for topical delivery in this study. For OVA vaccine delivery, lyophilized OVA/mannitol (1:25, weight/weight) powder was coated on adhesive patch surface as above and patches were cut into small pieces $\left(3 \times 3 \mathrm{~mm}^{2}\right)$, each containing $2.5 \mu \mathrm{g}$ OVA determined by BCA protein assay kit after extraction in PBS. For OVA vaccine delivery in the presence of NP(MPL), about $1.17 \mathrm{mg}$ OVA/NP(MPL) powder that contained $\sim 2.5 \mu \mathrm{g}$ OVA and $15 \mu \mathrm{g}$ MPL was weighed and coated on $3 \times 3 \mathrm{~mm}^{2}$ adhesive patch surface by spreading with a spatula. For Hib vaccine delivery, Hib vaccine powder containing $0.5 \mu \mathrm{g}$ Hib vaccine was weighed and coated on $3 \times 3 \mathrm{~mm}^{2}$ adhesive patch surface with spreading. For Hib vaccine delivery in 
the presence of NP(MPL), about $1.67 \mathrm{mg} \mathrm{Hib} / \mathrm{NP}(\mathrm{MPL})$ that contained $\sim 0.5 \mu \mathrm{g} \mathrm{Hib}$ vaccine and $15 \mu \mathrm{g}$ MPL was weighed and coated on $3 \times 3 \mathrm{~mm}^{2}$ adhesive patch surface.

\section{In vivo delivery}

Lateral back skin of adult or newborn mice were subjected to laser treatment to generate skin MCs followed by topical application of the above coated powder patches. Laser conditions used for OVA and Hib vaccination were $5 \mathrm{~mJ}$ energy and $10 \%$ coverage. Newborn mice were only explored for Hib vaccine delivery. Patches were covered with Tegaderm film to form an airtight system and further sealed in position with a narrow bandage without affecting free movement of mice. Patches were removed 2 days later in adult mice and 5 hours later in newborn mice. Newborn mice were kept on a digitally controlled heating pad at $37^{\circ} \mathrm{C}$ during the 5 -hour period and then returned to original cages. Immunization was repeated 2 weeks later. Serum anti-OVA or anti-Hib antibody titer was measured two weeks after boost.

\section{Serum antibody titer}

Small volume of blood $(\sim 20 \mu 1)$ was collected 2 weeks after boost. Serum anti-OVA or anti-Hib antibody titer was then measured by enzyme-linked immunosorbent assay (ELISA) by coating OVA $(10 \mu \mathrm{g} / \mathrm{ml})$ or Hib vaccine $(1 \mu \mathrm{g} / \mathrm{ml})$ into $96-w e l l$ ELISA plates at $4{ }^{\circ} \mathrm{C}$ overnight. After blocking in PBS supplemented in 5\% non-fat milk, diluted serum samples were added and incubated at room temperature for 2 hours. After washing, anti-mouse IgG, peroxidase linked species specific whole antibody (from sheep) were added (1:2500) and plates were kept at room temperature for 1 hour. After washing, TMB substrates were added and reactions were stopped by $2 \mathrm{M}$ $\mathrm{H}_{2} \mathrm{SO}_{4}$. Absorbance at $450 \mathrm{~nm}$ was read in a microplate reader (Molecular Device). 


\section{Delivery efficiency}

Patches were removed at different times and the remaining drugs were extracted into phosphate-balanced saline (PBS). Local skin surface was washed with PBS to recover undelivered drugs. Fluorescence intensity of SRB and TR-OVA in patch extracts before and after delivery, skin washes, and standard samples was measured at 565/586nm and 596/615nm, respectively, in Spectramax M2 Multi-Mode microplate reader (Molecular Devices). Concentrations of SRB and TR-OVA were calculated based on standard curves. Delivery efficiency was then calculated based on the formula: (patch coating amount-patch remaining amount-skin surface amount)/patch coating amount $\times 100 \%$. Drug amount on skin surface was found to be less than $1 \%$ in almost all deliveries (data not shown).

\section{PLGA NP preparation and characterization}

Single emulsion/solvent evaporation was used to prepare NP(SRB) and NP(MPL) [24]. In brief, $5 \mathrm{mg}$ SRB dissolved in $0.3 \mathrm{ml}$ dimethyl sulfoxide (DMSO) or $1 \mathrm{mg}$ MPL dissolved in $0.2 \mathrm{ml}$ chloroform was mixed with $80 \mathrm{mg}$ PLGA dissolved in $1.5 \mathrm{ml}$ dichloromethane (DCM). The mixture was added dropwise to 10ml 5\% PVA solution while sonicating at 12 Watts for 2 minutes to form oil/water emulsion. Solvent was evaporated at room temperature with continuous stirring for at least 4 hours. NP(SRB) and NP(MPL) were then pelleted and washed with distilled water for 3 times followed by lyophilization. NP size was measured by Zetasizer Nano ZS90 (Malvern). To quantify encapsulated drug amount, $\sim 1 \mathrm{mg}$ lyophilized NP(SRB) or NP(MPL) was weighed and hydrolyzed in $50 \mu 11 \mathrm{~N} \mathrm{NaOH}$ at $4^{\circ} \mathrm{C}$ overnight followed by adjusting $\mathrm{pH}$ to 7.4 with $1 \mathrm{~N} \mathrm{HCl}$. SRB amount was then quantified by measuring fluorescence 
intensity at $565 / 586 \mathrm{~nm}$. MPL amount was quantified by stimulation of dendritic cells to release cytokines following a published protocol [25]. Near 100\% encapsulation efficiency was observed for MPL (data not shown).

\section{Statistical analysis}

All values were expressed as Mean $\pm \mathrm{SEM}$ (standard error of mean). Student t-test was used to analyze the difference between mice groups. P value was calculated using PRISM software (GraphPad, San Diego, CA) and considered significant if it was less than 0.05 . 


\section{Results}

\section{Efficient full-surface delivery of power SRB}

We first explored whether full-surface-coated powder could have a good delivery via AFL-generated skin MCs. Readily detectable fluorescence SRB was explored first. We found $\sim 2.7 \mathrm{mg}$ SRB could be coated per $\mathrm{cm}^{2}$ of the patch. Lateral back skin of mice was exposed to laser at different energies $(2.5,5,10 \mathrm{~mJ})$ and percent coverages $(5,10,20 \%)$ followed by topical application of powder SRB-coated patches onto laser-treated skin. As shown in figure 1A-1C, increase of laser energy and percent coverage increased powder SRB delivery. Delivery efficiency reached over $90 \%$ in 8 hours for $2.5 \mathrm{~mJ}$ laser conditions (Figure 1A), in 4 hours for $5 \mathrm{~mJ}$ laser conditions (Figure 1B), and in 2 hours for 10mJ laser conditions (Figure 1C).
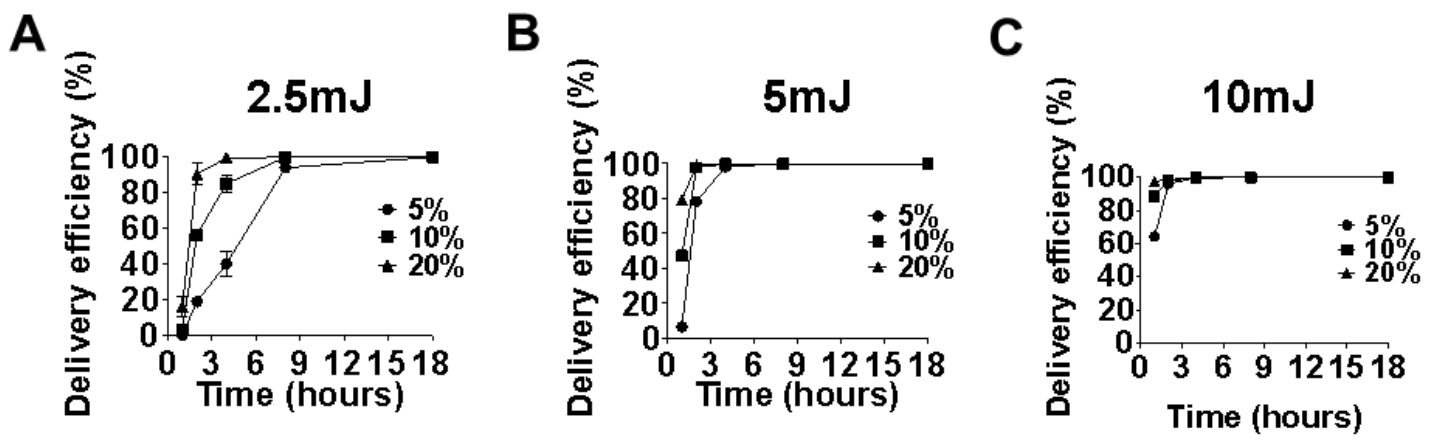

Figure 1. Efficient full-surface powder SRB delivery via laser-generated skin MCs

Female BALB/c mice were exposed to laser at different energies $2.5 \mathrm{~mJ}(\boldsymbol{A}), 5 \mathrm{~mJ}(\boldsymbol{B})$, and $10 \mathrm{~mJ}(\boldsymbol{C})$ and percent coverages $(5,10,20 \%)$. Powder SRB-coated patches were then topically applied. Patches were removed at indicated times (1, 2, 4, 8, 18 hours) and delivery efficiency was then quantified. $n=2-4$. 
To better understand dynamics of powder SRB delivery, patch images were taken at different times in laser $(5 \mathrm{~mJ} / 5 \%)$ group. Changes of SRB color from black in dry state to pink in wet state allowed us to explore temporospatial powder dissolution and delivery. As shown in figure 2, entire patch changed from black to dark pink color 1 hour after patch application, hinting the absorption of evaporated water by entire SRB powder. At the same time, we observed an array of pink-colored spots, matching skin MCs, hinting active delivery of dissolved SRB powder via skin MCs. Significant water absorption and active delivery was consistent with over $40 \%$ delivery efficiency at this time (Figure 2). Patch changed to strong pink color at 2 hours and the pink color was then gradually reduced after 4 hours (Figure 2). Our data indicated that water vapor could reach drug powder at least a few hundred microns away from skin MCs.

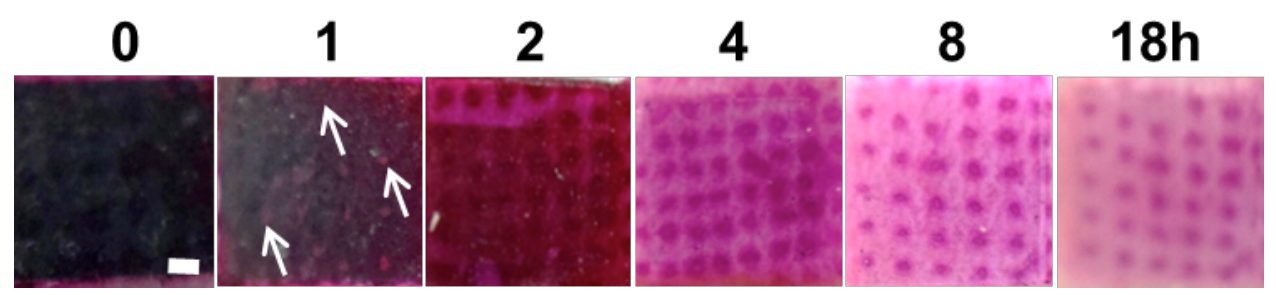

Figure 2. Imaging of SRB full-surface patch in vivo

Powder SRB-coated patches were topically applied onto laser (5mJ 5\%)-treated skin of BALB/c mice. Patch images were taken at different times. Experiments were repeated 3 times and representative patch images were shown. Arrows point to an array of pink colored spots matching skin MCs. Scale: $667 \mu m$.

Due to the importance of water evaporation in AFL-assisted powder delivery, we measured TEWL with Tewameter TM300 (Courage-Khazaka, Köln, Germany). We 
found laser ablation significantly increased TEWL of the skin even at the lowest laser condition (Figure 3A). Increase of laser energy or percent coverage accordingly increased TEWL (Figure 3A). Interestingly, we observed highly positive correlation between 1-hour delivery efficiency and TEWL (Figure 3B).
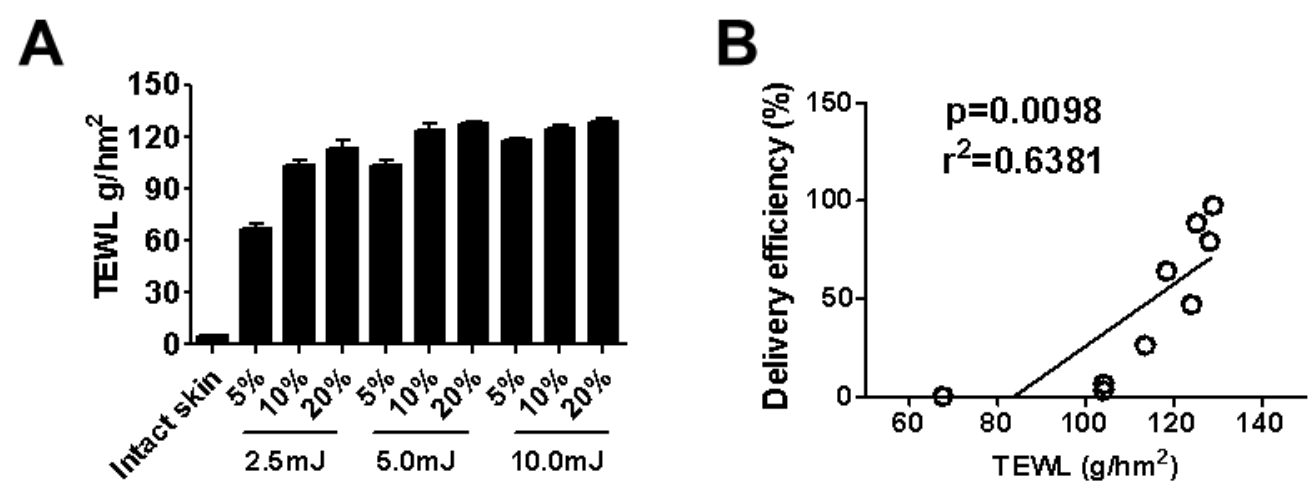

Figure 3. TEWL and correlation with powder SRB delivery efficiency

A. TEWL of laser-treated or non-treated control skin. $n=3$. B. Correlation analysis between 1-hour delivery efficiency and TEWL (9 data points).

\section{Efficient full-surface delivery of powder OVA}

Next we explored whether full-surface-coated powder model antigen ovalbumin (OVA) could be efficiently delivered via AFL-generated skin MCs. To facilitate detection, TR-OVA was mixed with OVA in a 1:8 weight ratio and then lyophilized and coated on full-surface of adhesive patches. We found $\sim 0.64 \mathrm{mg}$ OVA could be coated per $\mathrm{cm}^{2}$ of the patch. Delivery efficiency was explored by quantification and comparison of fluorescence intensity of patch-remained and total coated TR-OVA. As shown in figure $4 \mathrm{~A}-4 \mathrm{C}$, increase of laser energy and percent coverage was found to increase the rate of OVA delivery. Delivery efficiency reached more than $80 \%$ in 4 hours and more than $90 \%$ in 18 hours in most laser conditions. Interestingly, we still 
observed highly positive correlation between 1-hour delivery efficiency of OVA and TEWL (Figure 4D). This study hinted that full-surface-coated model antigen OVA could be efficiently delivered via laser-generated skin MCs.
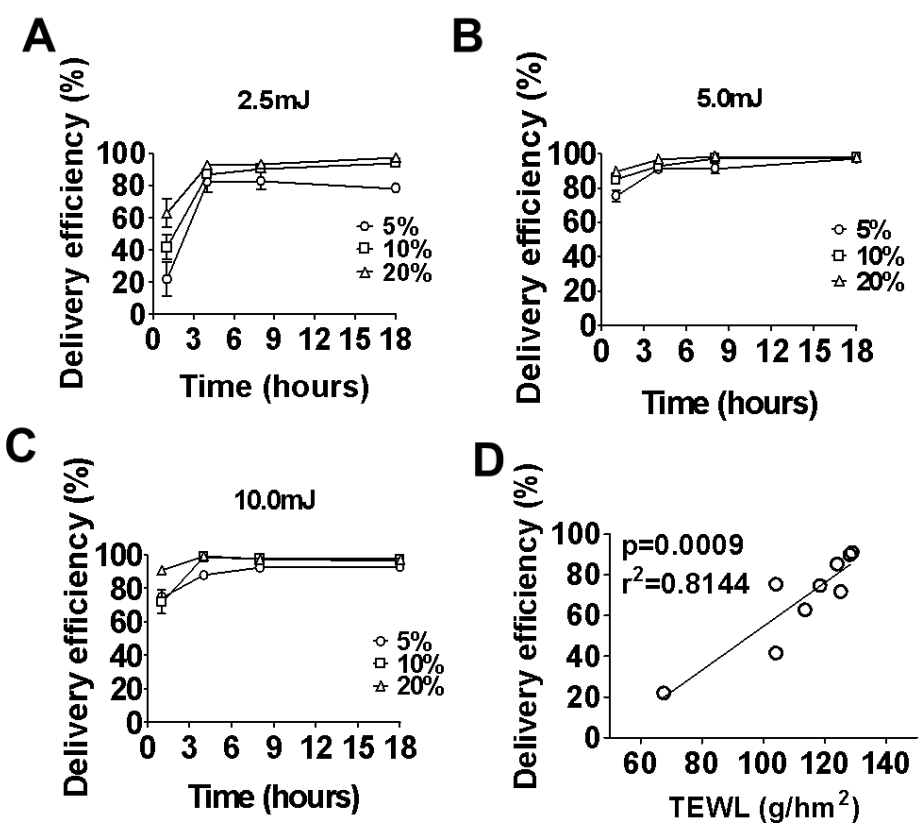

Figure 4. Efficient full-surface powder OVA delivery via laser-generated skin MCs A-C. Lateral back of BALB/c mice was exposed to laser followed by topical application of powder OVA-coated patches onto laser-treated skin. Patches were removed at different times $(1,4,8,18$ hours) and delivery efficiency was then quantified. $n=2-4$. D. Correlation analysis between 1-hour delivery efficiency and TEWL (9 data points).

\section{Efficient full-surface delivery of powder PLGA NPs}

To evaluate whether full-surface-coated powder PLGA NPs could be efficiently delivered via laser-generated skin MCs, SRB was encapsulated into PLGA NPs to facilitate the quantification of the delivery efficiency considering cargos were less 
likely to affect delivery efficiency. NP (SRB) was found to have a size of $254 \mathrm{~nm}$ with a polydispersity index (PDI) of 0.103 (Figure 5).

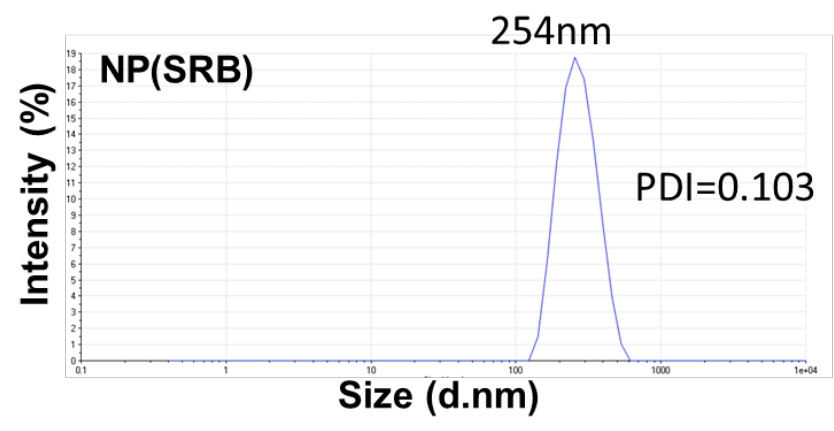

Figure 5. Size of NP(SRB) measured by Zetasizer Nano ZS90 (Malvern)

Lyophilized NP(SRB) (with approximately 1\% encapsulation efficiency of SRB) was then coated on adhesive patch surface and topically applied onto laser $(5 \mathrm{~mJ}$, 5/10/20\%)-treated skin of BALB/c mice. Delivery efficiency was measured 48 hours later.

SRB NP Delivery

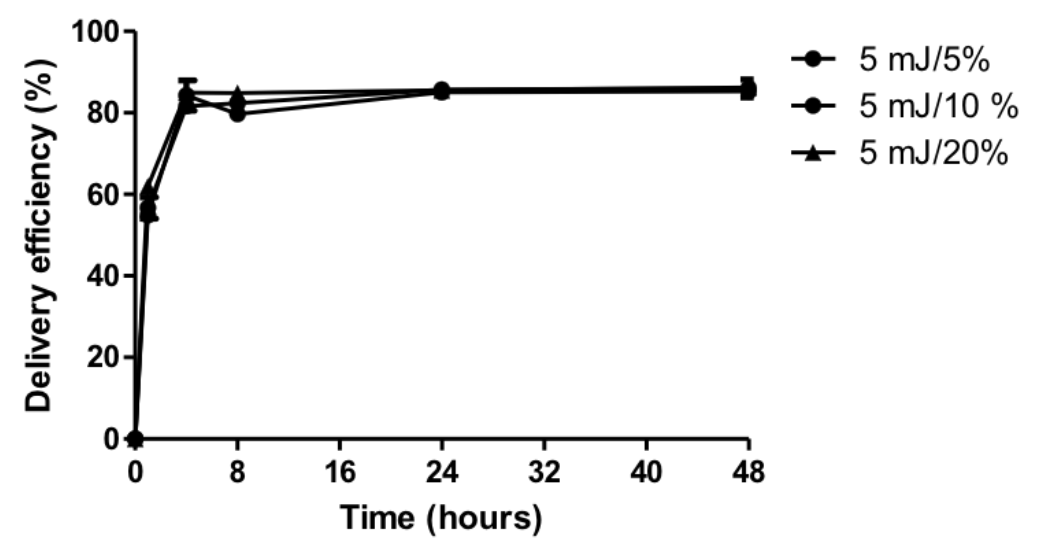

Figure 6. Efficient full-surface delivery of powder PLGA NPs

Lateral back skin of mice was exposed to AFL treatment at 5mJ energy and 5/10/20\% coverage followed by topical application of full-surface powder NP(SRB)-coated 
patches. Patches were removed at indicated times (1,4, 8, 24, 48 hours) and delivery efficiency was quantified. $n=3$.

As shown in Figure 6, more than $80 \%$ NP(SRB) was delivered into laser-treated skin within 4 hours, indicating AFL-assisted full-surface powder delivery could also efficiently deliver PLGA NPs. This study supported encapsulation of MPL into PLGA NPs for efficient delivery via laser-generated skin MCs.

\section{Laser-assisted full-surface powder OVA vaccination in the presence and absence of NP(MPL)}

Next we explored laser-assisted powder OVA immunization in the presence or absence of NP(MPL). NP(MPL) was found to have a size of $310 \mathrm{~nm}$ with a PDI of 0.264 (Figure 7). We also compared relative potency of laser-assisted powder delivery with needle-based SC delivery. The reason to choose SC delivery rather than ID delivery was to avoid significant local reactions following ID NP(MPL) delivery (data not shown). Powder OVA-coated patches with or without NP(MPL) were topically applied onto laser (5mJ/10\%)-treated skin of C57BL/6 mice in laser-based powder delivery (LPD). For comparison, the same dose of OVA or OVA/NP(MPL) were injected subcutaneously. Immunization was repeated 2 weeks later and serum antiOVA antibody titer was measured 2 weeks after prime and boost. Serum anti-OVA antibody titer after prime immunization was below 20 in OVA immunization alone groups and incorporation of NP(MPL) was found to increase serum anti-OVA antibody titer to 4000 in LPD group and 3400 in SC group (Left, Figure 8). After boost immunization, serum anti-OVA antibody titer increased to 300 in LPD of OVA alone and 220 in SC delivery of OVA alone (Right, Figure 8). 
Incorporation of NP(MPL) was found to increase serum antibody titer to 83,510 in LPD and 14,270 in SC delivery, respectively (Right, Figure 8), corresponding to 280-fold increase in LPD and 65-fold increase in SC delivery. Considering local reactions are a big concern for incorporation of adjuvants into ID or transdermal vaccinations, we further explored local safety of LPD of OVA in the presence of NP(MPL). As shown in Figure 9, minimal local reactions could be found in LPD of OVA alone and skin completely recovered in 1-2 days. Slight local reactions, like skin redness, could be found in LPD of OVA/NP(MPL) right after patch removal and skin completely recovered in 3 days (Figure 9). The most immunogenic LPD in the presence of NP(MPL) with good local safety supported further exploration of this technology for real vaccine delivery.

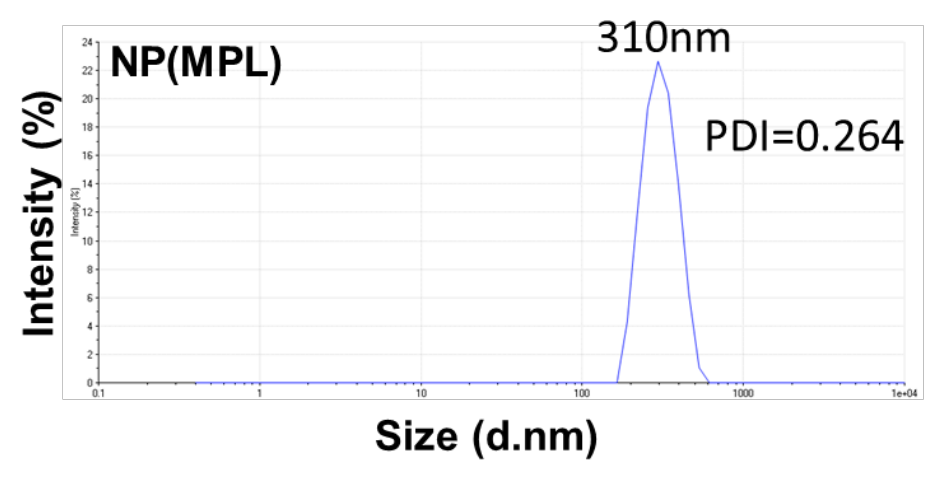

Figure 7. Size of NP(MPL) measured by Zetasizer Nano ZS90 (Malvern) 

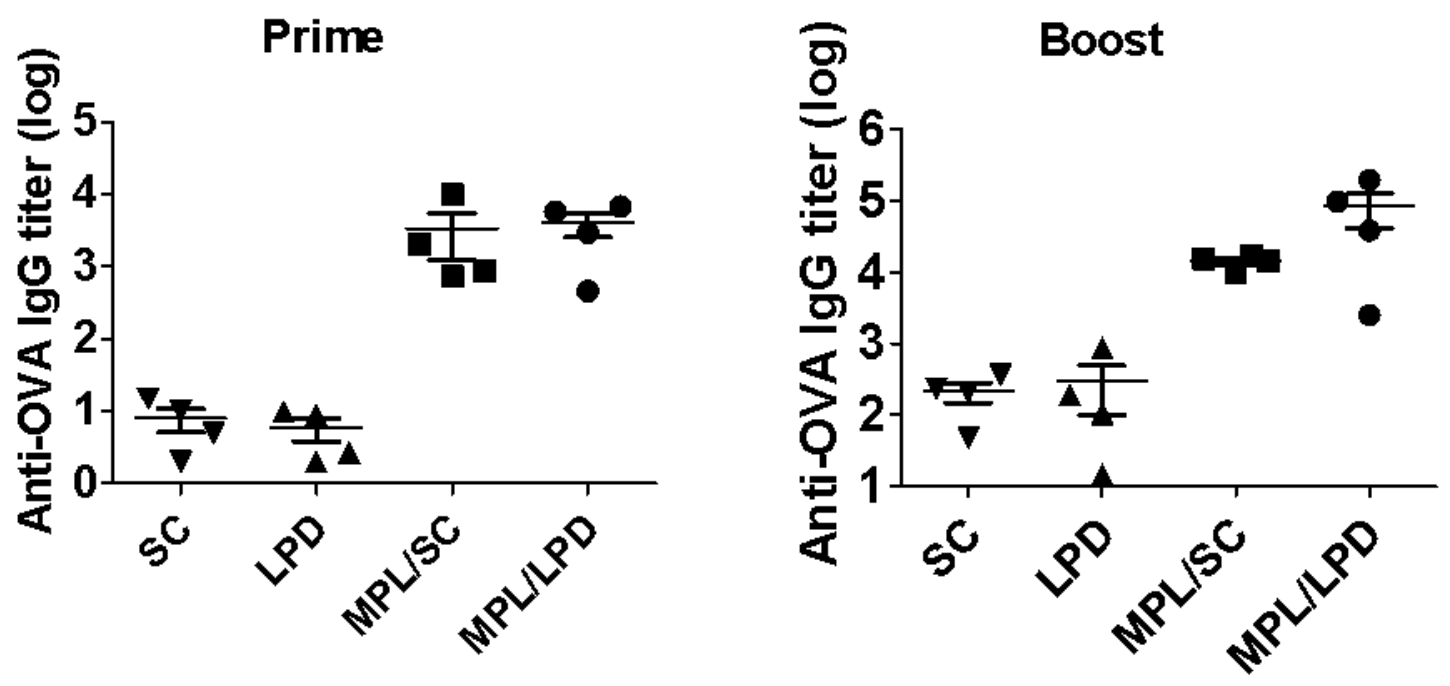

Figure 8. Comparison of laser-based powder OVA delivery with subcutaneous (SC) delivery in the presence and absence of $N P(M P L)$

Powder OVA vaccine-coated patches with or without NP(MPL) were topically applied onto laser (5mJ/10\%)-treated skin of C57BL/6 mice. In other group, same dose of OVA or OVA/NP(MPL) were injected subcutaneously. Patches were removed 48 hours later. Immunizations were repeated two weeks later and serum anti-OVA antibody titer was measured 2 weeks after prime and boost. $n=4$.

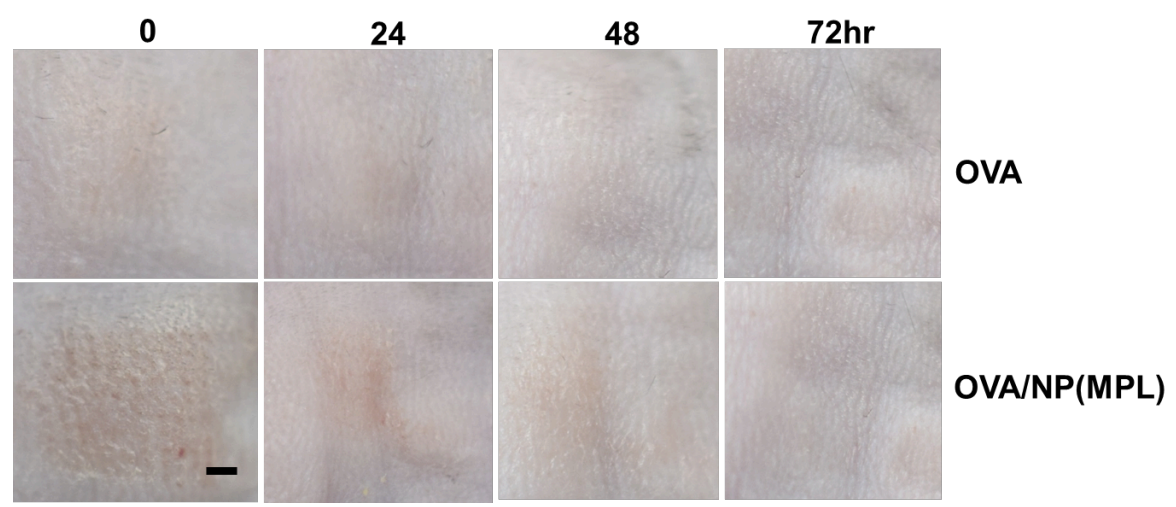

Figure 9. Minimal local reactions following LPD of $O V A$ in the presence or absence of NP(MPL) 
Lateral back skin of mice was exposed to laser (5mJ/10\%) followed by topical application of powder OVA-coated patches $(3 \times 3 \mathrm{~mm} 2)$ or powder OVA/NP $(M P L)$ patches $(5 \times 5 \mathrm{~mm} 2)$. Skin images were taken right after patch removal and daily for 3 days after patch removal to explore local reactions in the presence or absence of $N P(M P L)$ adjuvant. Scale: $1 \mathrm{~mm}$.

\section{Laser-assisted full-surface powder Hib vaccination in the presence or absence of NP(MPL) in newborn and adult mice}

Hib vaccines are currently given to infants 3 times within the first 6 months of life by needle injection to protect serious illnesses caused by the bacteria Haemophilus Influenzae. The needle-free and potentially painless LPD may provide a better immunization strategy for newborns. In addition, incorporation of MPL adjuvant may improve Hib vaccine immunogenicity. To explore this, powder Hib vaccine-coated patches in the presence or absence of NP(MPL) were topically applied onto laser $(5 \mathrm{~mJ} / 10 \%)$-treated skin of newborn and adult mice. Immunization was repeated 2 weeks later. As shown in figure 10A, LPD and SC delivery of Hib vaccine alone in newborn mice induced weak immune responses with serum anti-Hib vaccine antibody titer below 100. Simultaneous delivery of NP(MPL) in newborn mice significantly increased serum anti-Hib vaccine antibody titer to 2,520 in SC group and 1,810 in LPD group (Figure 10A). Due to the mature immune system, the same immunization induced much higher anti-Hib vaccine antibody titer in adult mice. As shown in figure 10B, Hib vaccination alone induced serum anti-Hib vaccine antibody titer of 200 and 730 in SC and LPD group, respectively. Co-delivery of NP(MPL) increased serum 
antibody titer to 17,630 and 11,310 in SC and LPD group, respectively (Figure 10B), corresponding to 88 - and 15 -fold increase, respectively. These results validated LPD for Hib vaccine delivery and the effectiveness of NP(MPL) to boost Hib vaccination in newborn and adult mice.
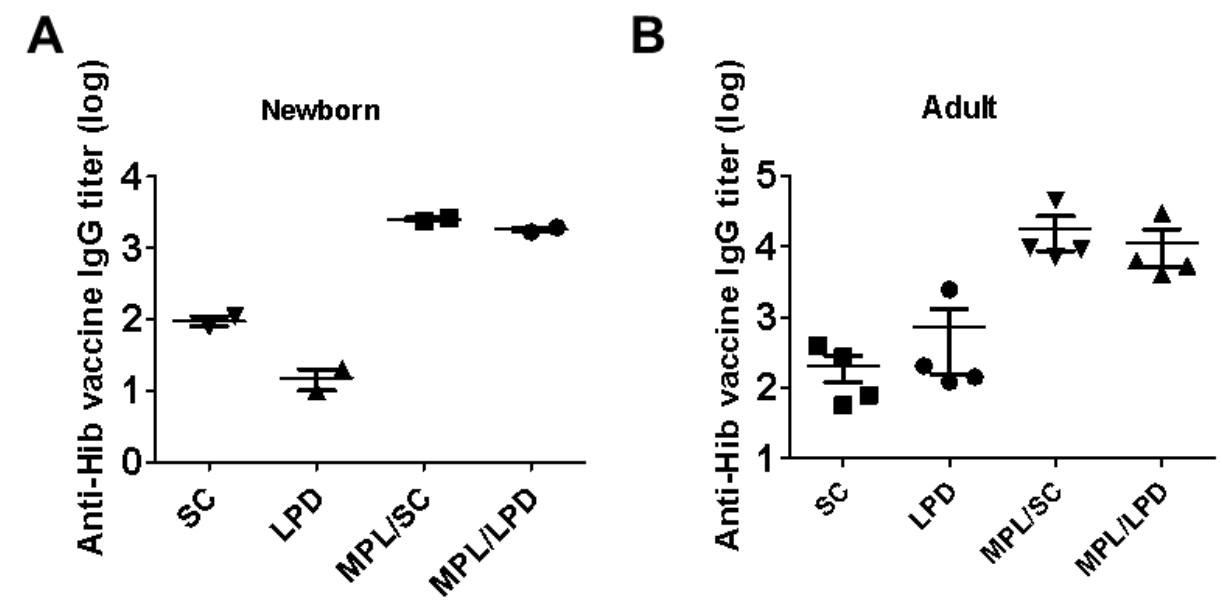

Figure 10. Comparison of laser-based powder Hib vaccine delivery with SC delivery in the presence or absence of $N P(M P L)$

Powder Hib vaccine-coated patches with or without NP(MPL) were topically applied onto laser (5mJ/10\%)-treated skin of C57BL/6 mice. In SC group, the same dose of Hib vaccine with or without NP(MPL) were subcutaneously injected in newborn (A) and adult mice (B). Patches were removed 48 hours later. Immunizations were repeated two weeks later and serum anti-Hib vaccine antibody titer was measured 2 weeks after prime and boost. $n=2$ in newborn mice 4 in adult mice.

\section{Efficient full-surface powder OVA delivery in guinea pigs}

Due to the thin mouse skin, laser-assisted full-surface powder delivery was further explored in guinea pigs with a similar skin to human skin in anatomy and immunology 
[27]. As in mouse study, TR-OVA was mixed with OVA at 1:8 ratio and then lyophilized and coated on full-surface adhesive patch surface and then topically applied onto laser-treated skin of guinea pigs. We found the same laser condition generated lower TEWL in guinea pig skin (Figure 11) as compared to mouse skin (Figure 3A).

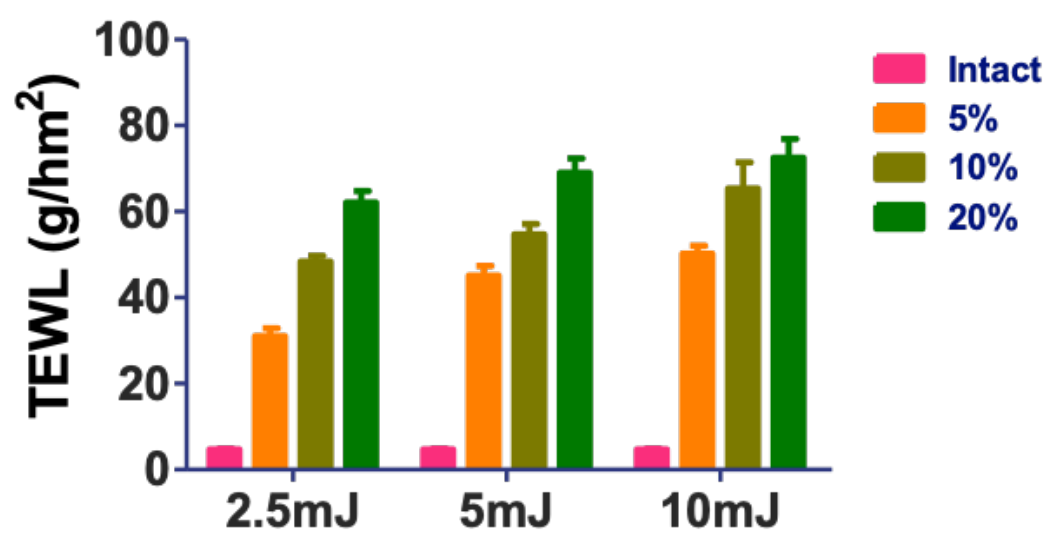

Figure 11. TEWL of laser-treated guinea pig skin

Hairless guinea pigs were treated with laser at different combinations of laser energy $(2.5,5,10 \mathrm{~mJ})$ and percent coverage $(5,10,20 \%)$. TEWL of intact skin and lasertreated skin was measured right after treatment. $n=3$.

Therefore, two pulses of laser at relatively high laser energies (10 and $20 \mathrm{~mJ})$ and $10 \%$ coverage were used to treat skin to generate skin MCs to explore the delivery efficiency in guinea pigs. Patches were removed 24 hours later and as shown in Figure 12, both laser conditions elicited almost complete delivery of TR-OVA/OVA mixture. This study indicated that laser-based full-surface powder vaccination was also promising to elicit potent immune responses in guinea pigs with a similar skin to human skin. 


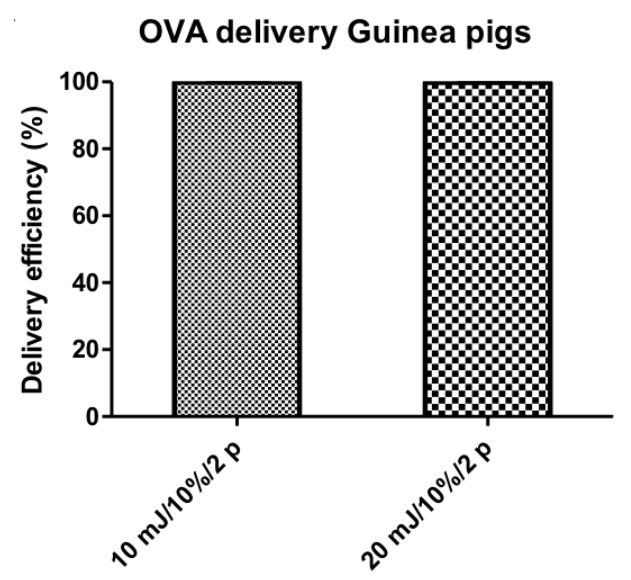

Figure 12. Efficient delivery of powder OVA in guinea pigs

Lateral back skin of hairless guinea pigs was exposed to laser at indicated conditions followed by topical application of powder TR-OVA/OVA-coated patches onto lasertreated skin. Patches were removed at 24 hours and delivery efficiency was then quantified. $n=3$. 


\section{Discussion}

This study explored laser-based full-surface powder delivery (LPD) for needle-free transdermal vaccination. LPD is based on coating powder vaccines on full-surface adhesive patches followed by topical application powder vaccine-coated patches onto laser-generated skin MCs to deliver vaccines into the skin. We found full surfacecoated powder SRB, model antigen OVA, and PLGA NPs could be efficiently delivered into the skin via laser-generated MCs in murine models. More than $80 \%$ delivery efficiency of SRB, OVA, and NP(SRB) could be achieved within 4 hours (Figure 1A-1C, 4A-4C \& 6). Our studies indicated that water evaporated from skin MCs was highly competent to dissolve full surface-coated small chemicals and macromolecules and elicit high-efficient delivery via laser-generated skin MCs. Remarkably PLGA NPs could also achieve high-efficient delivery via laser-generated skin MCs. We also found crucial roles of water evaporation in laser-based full-surface powder vaccination as supported by a highly positive correlation between early-stage delivery efficiency and TEWL value of the skin (Figure 3B \& 4D).

LPD of OVA was found to induce comparable immune responses to SC delivery (Figure 8). Incorporation of NP(MPL) was found to significantly boost OVA immunization in both deliveries (Figure 8). LPD of OVA in the presence of NP(MPL) induced $\sim 6$ times higher anti-OVA antibody titer than SC delivery (Figure 8 ). LPD of OVA in the presence of NP(MPL) induced minimal local reactions (Figure 9). This is very important considering SC or transdermal delivery of vaccine alone was found to induce more frequent and severe local reactions as compared to IM delivery [28,29], 
which hampers the incorporation of external adjuvants to further boost ID or transdermal vaccination.

LPD of Hib vaccine was found to also induce comparable immune responses to SC delivery in adult mice (Figure 10B). Incorporation of NP(MPL) was found to further boost Hib vaccination in both deliveries (Figure 10B). Besides significantly boosting vaccination, no significant difference of anti-Hib vaccine antibody titer could be found between LPD and SC groups in adult mice. In newborn mice, LPD of Hib vaccine also induced comparable immune responses to SC delivery (Figure 10A). Furthermore, codelivery of NP(MPL) also significantly increased anti-Hib vaccine antibody titer in newborn mice (Figure 10A). Our studies validated LPD for transdermal vaccine delivery and NP(MPL) as a highly potent adjuvant to further boost vaccination. In these studies, we observed weaker immune responses induced in newborn mice as compared to adult mice, which can be explained by the immature immune system of newborn mice [30]. To our knowledge, this study represented one of the two studies that successfully delivered MPL via the transdermal route. In the other study, MPL was formulated with trehalose into particulate form for ID ballistic delivery by PowderJect [31]. In PowderJect delivery, vaccines needed to be formulated with heavy metals for successful deposition into the skin. LPD involves no complex powder formulation and can efficiently deliver vaccine into the skin with ease. Our study also found high efficient delivery of full surface-coated powder OVA via laser-treated skin in guinea pigs. This study supports the potential application of laser-based full-surface powder vaccination in humans. Direct powder vaccination might also eliminate coldchain storage, which is crucial for vaccination in resource-poor countries. 


\section{Conclusion}

In summary, we developed laser-based full-surface powder delivery as an innovative delivery platform for transdermal vaccination. Laser-based full-surface powder vaccine delivery could elicit comparable immune responses to needle-based SC delivery. Such a delivery platform could also efficiently deliver NP(MPL) and codelivery of NP(MPL) was found to profoundly boost OVA and Hib vaccination in both newborn and adult mice. Laser-based full-surface powder vaccination in the presence of NP(MPL) also induced mild local reactions and laser-treated skin could recover within 3 days. Laser-based full-surface powder delivery was found to also efficiently deliver OVA in guinea pigs with a similar skin to human skin. The safe and highly immunogenic laser-based full-surface powder vaccination warrants further investigation for human vaccination use. 


\section{Acknowledgements}

This work is partly supported by the National Institutes of Health grants DA033371 and AI107678 (to X.Y.C.). Microplate reader used in this work is supported by an Institutional Development Award (IDeA) from the National Institute of General Medical Sciences of the National Institutes of Health grant P20GM103430. 


\section{References}

[1] R.K. Tyagi, N.K. Garg, R. Jadon, T. Sahu, et al. Elastic liposome-mediated transdermal immunization enhanced the immunogenicity of P. falciparum surface antigen: MSP-119. Vaccine, 33 (36) (2015), pp. 4630-4638.

[2] W.H. Kong, D.K. Sung, H. Kim, J.A. Yang, et al. Self-adjuvanted hyaluronateantigenic peptide conjugate for transdermal treatment of muscular dystrophy. Biomaterials, 81 (2016), pp. 93-103.

[3] K. Matsuo, S. Hirobe, N. Okada, S. Nakagawa. Frontiers of transcutaneous vaccination systems: novel technologies and devices for vaccine delivery. Vaccine, 31 (19) (2013), pp. 2403-2415.

[4] Cook, L. S. (2016) Needle phobia. Journal of Infusion Nursing 39(5): 273-279.

[5] S. Marshall, L.J. Sahm, A.C. Moore. The success of microneedle-mediated vaccine delivery into skin. Hum. Vaccin. Immunother. (2016).

[6] A.M. Römgens, D.L. Bader, J.A. Bouwstra, C.W.J. Oomens. A theoretical compartment model for antigen kinetics in the skin. Eur. J. Pharm. Sci., 84 (2016), pp. 18-25.

[7] L. Adam, P. Rosenbaum, A. Cosma, R. Le Grand, et al. Identification of skin immune cells in non-human primates. J. Immunol. Methods, 426 (2015), pp. 42-49.

[8] Banchereau J, Steinman RM. Dendritic cells and the control of immunity. Nature 1998:392.

[9] Kenney RT, Frech SA, Muenz LR, Villar CP, Glenn GM. Dose sparing with intradermal injection of influenza vaccine. N Engl J Med 2004;351:2295-301. 
[10] Zhang, L., Wang, W., \& Wang, S. (2015). Effect of vaccine administration modality on immunogenicity and efficacy. Expert review of vaccines, 14(11), 150923.

[11] Charest AF, McDougall J, Goldstein MB. A randomized comparison of intradermal and intramuscular vaccination against hepatitis B virus in incident chronic hemodialysis patients. Am J Kidney Dis. 2000;36(5):976-82.

[12] Levin, Y., Kochba, E., Hung, I., \& Kenney, R. (2015). Intradermal vaccination using the novel microneedle device MicronJet600: Past, present, and future. Human vaccines \& immunotherapeutics, 11(4), 991-7.

[13] Beran, J., Ambrozaitis, A., Laiskonis, A., Mickuviene, N., Bacart, P., Calozet, Y., Demanet, E., Heijmans, S., Van Belle, P., Weber, F., ... Salamand, C. (2009). Intradermal influenza vaccination of healthy adults using a new microinjection system: a 3-year randomised controlled safety and immunogenicity trial. BMC medicine, $7,13$.

[14] Logomasini MA, Stout RR, Marcinkoski R. Jet injection devices for the needlefree administration of compounds, vaccines, and other agents. Int J Pharm Compd. 2013 Jul-Aug;17(4):270-80.

[15] Kim Y-C, Quan F-S, Yoo D-G, Compans RW, Kang S-M, Prausnitz MR. Improved influenza vaccination in the skin using vaccine coated microneedles. Vaccine 2009;27:6932-8.

[16] Kim Y-C, Quan F-S, Yoo D-G, Compans RW, Kang S-M, Prausnitz MR. Enhanced memory responses to seasonal H1N1 influenza vaccination of the skin with the use of vaccine-coated microneedles. J Infect Dis 2010;201:190-8. 
[17] N.G. Rouphael, M. Paine, R. Mosley, S. Henry, D.V. McAllister, H. Kalluri, et al. The safety, immunogenicity, and acceptability of inactivated influenza vaccine delivered by microneedle patch (TIV-MNP 2015): a randomised, partly blinded, placebo-controlled, phase 1 trial. Lancet, 390 (2017), pp. 649-658.

[18] M. Haedersdal, F.H. Sakamoto, W.A. Farinelli, A.G. Doukas, J. Tam, R.R. Anderson, Fractional CO(2) laser-assisted drug delivery, Lasers Surg. Med. 42 (2010) 113-122.

[19] Y. Cao, P. Kakar, M.N. Hossen, M.X. Wu, X. Chen, Sustained epidermal powder drug delivery via skin microchannels, Journal of controlled release : official journal of the Controlled Release Society, 249 (2017) 94-102.

[20] Lee, Pan, Wang, Zhuo, Huang, and Fang. "Erbium:YAG Laser Enhances Transdermal Peptide Delivery and Skin Vaccination." Journal of Controlled Release 128.3 (2008): 200-08.

[21] Hessenberger, M., Weiss, R., Weinberger, E. E., Boehler, C., Thalhamer, J., \& Scheiblhofer, S. (2013). Transcutaneous delivery of CpG-adjuvanted allergen via laser-generated micropores. Vaccine, 31(34), 3427-34.

[22] Chen, Kositratna, Zhou, Manstein, and Wu. "Micro-fractional Epidermal Powder Delivery for Improved Skin Vaccination." Journal of Controlled Release 192 (2014): 310-16.

[23] S. Lee, M.T. Nguyen, Recent advances of vaccine adjuvants for infectious diseases, Immune network, 15 (2015) 51-57. 
[24] R.L. McCall, R.W. Sirianni, PLGA nanoparticles formed by single- or doubleemulsion with vitamin E-TPGS, Journal of visualized experiments : JoVE, (2013) 51015.

[25] S.P. Kasturi, I. Skountzou, R.A. Albrecht, D. Koutsonanos, T. Hua, H.I. Nakaya, R. Ravindran, S. Stewart, M. Alam, M. Kwissa, F. Villinger, N. Murthy, J. Steel, J. Jacob, R.J. Hogan, A. Garcia-Sastre, R. Compans, B. Pulendran, Programming the magnitude and persistence of antibody responses with innate immunity, Nature, 470 (2011) 543-547.

[26] Mahmoudi, S., \& Keshavarz, H. (2017). Efficacy of phase 3 trial of RTS, S/AS01 malaria vaccine: The need for an alternative development plan. Human vaccines \& immunotherapeutics, 13(9), 2098-2101.

[27] Sueki H, Gammal C, Kudoh K, Kligman AM. 2000. Hairless guinea pig skin: anatomical basis for studies of cutaneous biology. Eur J Dermatol 10:357-364. [28] Jeena PM, Chhagan MK, Topley J, Coovadia HM. Safety of the intradermal Copenhagen 1331 BCG vaccine in neonates in Durban, South Africa. Bull. World Health Organ. 2001;79:337-343.

[29] Leroux-Roels I, Vets E, Freese R, Seiberling M, Weber F, Salamand C, LerouxRoels G. Seasonal influenza vaccine delivered by intradermal microinjection: A randomised controlled safety and immunogenicity trial in adults. Vaccine. 2008;26:6614-6619.

[30] A. Saso, B. Kampmann, Vaccine responses in newborns, Seminars in immunopathology, 39 (2017) 627-642. 
[31] D. Sarphie, W.F. Swain, G.J. Widera, R.J. Drape, D. Chen, Transdermal delivery of particulate vaccine compositions, in, Powderject Vaccines, Inc., US, 1999. 


\title{
MANUSCRIPT III
}

\author{
Laser-assisted hydrogel-based drug delivery \\ Prateek Kakar and Xinyuan Chen
}

(Prepared for submission to International Journal of Pharmaceutics) 


\section{Laser-assisted hydrogel-based drug delivery \\ Prateek Kakar and Xinyuan Chen ${ }^{*}$}

Biomedical \& Pharmaceutical Sciences, College of Pharmacy, University of Rhode Island, Kingston, RI

*Address Correspondence to: Xinyuan Chen, Biomedical \& Pharmaceutical Sciences, College of Pharmacy, University of Rhode Island, Kingston, RI 02881,TEL: 401-8745033; FAX: 401-874-5787; xchen14@uri.edu

The authors have no conflict of interest to declare. 


\begin{abstract}
Transdermal delivery is highly attractive for alternative drug delivery and yet is hampered by the barrier function of the stratum corneum (SC) layer of the skin. Ablative fractional laser (AFL) provides a safe and efficient approach to ablate SC layer to facilitate transdermal drug delivery. Different dosage forms like liquids, cream, lotion, and powder have been found to efficiently deliver via AFL-generated skin microchannels (MCs). Hydrogel-mediated drug delivery has recently received extensive attention due to its porosity, biocompatibility, and biodegradability. This study explored whether hydrophilic drugs could be encapsulated into hydrogels for efficient delivery via AFL-generated skin MCs. We found poly (vinyl alcohol) (PVA) but not poly 2-Hydroxyethyl methacrylate (pHEMA) hydrogels to elicit sustained delivery via AFL-generated skin MCs in murine models.
\end{abstract}

Keywords: hydrogel; transdermal drug delivery; laser; polyvinyl alcohol. 


\section{Introduction}

Oral drug delivery often requires frequent dosing or high drug amounts to achieve therapeutic effects. This can lead to poor patient compliance and drug toxicity $[1,2]$. Oral delivery also leads to poor bioavailability of certain drugs due to extensive degradation and metabolism in the gastrointestinal systems. Transdermal delivery is highly attractive for alternative drug delivery and is yet hampered by the barrier function of the stratum corneum (SC) layer of the skin [3, 4].

Ablative fractional laser (AFL) has been explored as a safe and efficient approach to ablate SC layer to facilitate transdermal drug delivery in different dosage forms [5]. In one study, successful permeation of diclofenac in solution form was achieved in excised porcine and human skin using a portable AFL named Precise Lasers Epidermal System (P.L.E.A.S.E. ${ }^{\circledR}$ ) [6]. In another study, a topical terbinafine antifungal cream was administered to patients with onychomycosis with a carbondioxide AFL [7]. In our previous studies, we efficiently delivered powdered AZT via AFL-generated skin microchannels (MCs) over a span of 15 hours with a $100 \%$ bioavailability enhancement as compared to oral delivery in mouse models [8]. Hydrogels are 3-dimensional cross-linked water-soluble polymer networks and can be prepared in different physical forms, like slabs, films, and coatings. Hydrogels are highly biocompatible and biodegradable and drugs can be loaded into the gel matrix for slow diffusion out of the gel. Hydrogels can also be developed to respond to external stimuli to release drugs. Hydrogels can also adapt to the shape of the biological surfaces that they are applied to and are attractive to develop mucoadhesive 
drug delivery systems. Hydrogels also have tunable mechanical strength based on concentration of polymers [9].

Hydrogel drug delivery systems have been developed for controlled drug release. Hydrogels can be prepared from synthetic polymers such as poly (vinyl alcohol) (PVA) [10], or natural polymers such as alginate [11], collagen [12] and chitosan [13]. In situ gelling hydrogels are injected in liquid form and follow a sol-gel transition in the body. Insulin release was observed over two weeks in an in situ gelling hydrogel in mice [14]. In other cases, hydrogels need to be surgically implanted, which limits its routine practice in drug delivery applications [15-17].

Here we explored whether topical application of drug-loaded poly 2-Hydroxyethyl methacrylate (pHEMA) and PVA hydrogels onto AFL-treated skin could elicit highefficient drug delivery in murine models. We found PVA but not pHEMA hydrogel systems elicited efficient and sustained SRB and AZT delivery via AFL-generated skin MCs. 


\section{Materials and methods}

\section{Reagents}

Sulforhodamine B (SRB, 230161), poly(vinyl alcohol) (PVA, 563900, 1,30,000 Da), 2-2'-Azobis(2-methylpropionitrile) (AIBN,441090), dichloromethylsilane (440248)

and zidovudine (AZT, A2169) were purchased from Sigma (St. Louis, MO). 3'-Azido3'-deoxythymidine (AZT-IS, MG103), AZT internal standard, was purchased from Moravek Biochemicals (Brea, CA). Ethylene dimethacrylate (EGDMA, 409922500), 2-Hydroxyethyl methacrylate (HEMA, 156335000) and N-Vinyl-2-pyrrolidone (NVP, 140925000) were purchased from Acros Organics.

\section{pHEMA hydrogel preparation}

pHEMA hydrogels were prepared as per a published protocol [18]. In brief, $0.089 \mathrm{~g}$ Vinyl-2-pyrrolidone (VP) as co-monomer and $0.127 \mathrm{~g}$ Ethylene dimethacrylate (EGDMA) as a cross-linker was added to $8 \mathrm{ml}$ 2-Hydroxyethyl methacrylate (HEMA) (monomer) followed by the addition of the initiator 2-2'-Azobis(2methylpropionitrile) (AIBN) (0.013 g) and continuous stirring until a clear solution was obtained. This solution was poured between dichloromethylsilane-treated glass plates and incubated at $50^{\circ} \mathrm{C}$ for 12 hours followed by $70^{\circ} \mathrm{C}$ for 24 hours. The plates were then removed and immersed in distilled water until the hydrogel separated. The hydrogel was then immersed in distilled water for one week to remove unreacted monomers. Water was changed once a day.

SRB loading was carried out by immersing the hydrogels in $50 \mathrm{mg} / \mathrm{ml}$ solution of SRB for 5 days. The hydrogels were stored at $4^{\circ} \mathrm{C}$ until further use. 


\section{PVA hydrogel preparation}

PVA hydrogels were prepared by $5 \% \mathrm{w} / \mathrm{v}$ high molecular weight PVA in water. Freeze thaw method was used to prepare hydrogels. Drugs were loaded into the polymer solution and stirred for 5 hours. The solution was then transferred to plastic petri dishes and freeze-thawed for 3 cycles. Each freezing cycle was 2 hours at $-20^{\circ} \mathrm{C}$ followed by thawing for 1 hour at room temperature. The obtained hydrogels were cut to required size, sealed in plastic bags and stored at $4^{\circ} \mathrm{C}$ until further use.

\section{Animals}

BALB/c mice (female, 6-8 weeks old) were purchased from Charles River Laboratories (Wilmington, MA). They were housed and maintained in the animal quarters of University of Rhode Island. For the experiments, the animals were anaesthetized for hair removal, laser treatment, patch application and patch removal. For in vitro study, mice were euthanized after laser treatment with $\mathrm{CO}_{2}$ and skin was dissected. Institutional Animal Care and Use Committees of URI approved all procedures.

\section{Generation of skin MCs}

An UltraPulse Fractional $\mathrm{CO}_{2}$ Laser purchased from Lumenis Inc. (San Jose, CA) was used to generate skin MCs.

\section{Patch application}

Hairless dorsal mouse skin was exposed to laser treatment followed by careful placement of the patches and then a thin $3 \mathrm{M}$ Tegaderm membrane to completely cover the patches and to seal the system. Patches were gently pressed to ensure a close 
contact with the skin. A bandage was applied over the delivery area to ensure that the patches stay in position till removal.

\section{Delivery efficiency and patch extraction}

Patches were removed at different times and the remaining drugs were extracted into phosphate-balanced saline (PBS). The patches were immersed in PBS for 7 days and agitated on a vortex several times a day to expedite the release of remaining drugs. Local skin surface was washed with PBS to recover undelivered drugs. Fluorescence intensity of SRB in patch extracts before and after delivery, skin washes, and standard samples were measured at 565/586nm, in Spectramax M2 Multi-Mode microplate reader (Molecular Devices). Concentrations of SRB were calculated based on standard curves. Delivery efficiency was then calculated based on the formula: (patch coating amount-patch remaining amount-skin surface amount)/patch coating amount $\times 100 \%$. AZT concentrations were measured using LC-MS/MS as described in later section.

\section{Franz cell studies}

Franz cell system with orifice diameter of $5 \mathrm{~mm}$ and recipient chamber volume of 1.5 $\mathrm{ml}$ were custom made by Permegear. Patches were applied to laser treated skin and excised. It was then mounted on top of the recipient chamber and secured in place by a $3 \mathrm{M}$ tegaderm membrane. Donor chamber was placed above this and both the chambers were clamped. $1.5 \mathrm{ml}$ PBS was added to the recipient chamber and a small stir bar ensured continuous stirring of the solution. $100 \mu 1$ samples were collected at various time points and replenished with an equal volume of fresh PBS. The Franz cells were placed on a magnetic stir plate which was maintained at $37^{\circ} \mathrm{C}$ throughout the study. 


\section{LC-MS/MS quantification of AZT}

Liquid chromatography-tandem mass spectrometry (LC-MS/MS) was employed to quantify blood AZT levels [19]. Patch extracts and AZT standards $(6.25,12.5,25,50$, 100, 200, $400 \mathrm{ng} / \mathrm{ml}$ ) were mixed with $100 \mathrm{ng} / \mathrm{ml}$ AZT-Internal standard (IS) Serum samples were diluted 20 times, mixed with $100 \mathrm{ng} / \mathrm{ml}$ AZT-IS and filtered through 10kDa cutoff Amicon filter purchased from EMD Millipore (Burlington, MA) Samples were loaded into an AB Sciex 4500 QTRAP LC-MS/MS equipped with Shimadzu LC-20AD pumps and a QTRAP 4500 System. A Synergi Hydro-RP 80A, $2.0 \times 150 \mathrm{~mm}, 4 \mu \mathrm{m}$ particle size analytical column (Phenomonex, Torrance, $\mathrm{CA}$ ) was used for sample separation. Acquisition was performed in multiple reactionmonitoring (MRM) mode using m/z 268/127 for AZT and 271/130 for AZT-IS detection. A standard curve was generated by plotting peak area ratios of AZT to AZT-IS against AZT concentrations and used to quantify AZT levels in the unknown samples.

\section{Statistical analysis}

All values were expressed as Mean \pm SEM (standard error of mean). Student t-test was used to analyze the difference between mice groups. P value was calculated using PRISM software (GraphPad, San Diego, CA) and considered significant if it was less than 0.05 . 


\section{Results}

\section{In vitro SRB delivery via pHEMA hydrogel}

Pre-cut hydrogels were immersed in a $50 \mathrm{mg} / \mathrm{ml}$ solution of SRB for 5 days. We found $225 \mu \mathrm{g}$ SRB could be loaded in a $4 \times 4 \mathrm{~mm}^{2}$ patch with $1 \mathrm{~mm}$ thickness. Lateral back skin of mice was exposed to laser $(5 \mathrm{~mJ} / 5 \%)$ followed by dissection and mounting onto the recipient chamber of the Franz diffusion cell. SRB-incorporated hydrogels of $4 \times 4$ $\mathrm{mm}^{2}$ in size were then topically applied and secured with a $3 \mathrm{M}$ tegaderm membrane. $100 \mu \mathrm{l}$ samples were collected at multiple intervals for 30 days and replenished with $100 \mu l$ fresh PBS.

SRB amount in harvested samples and patches were analyzed 30 days after delivery. We observed a linear delivery of SRB in the first 15 days followed by cessation of delivery in the next 15 days (Figure 1A). Delivery efficiency reached about $60 \%$ at day 30 (Figure 1B).

A

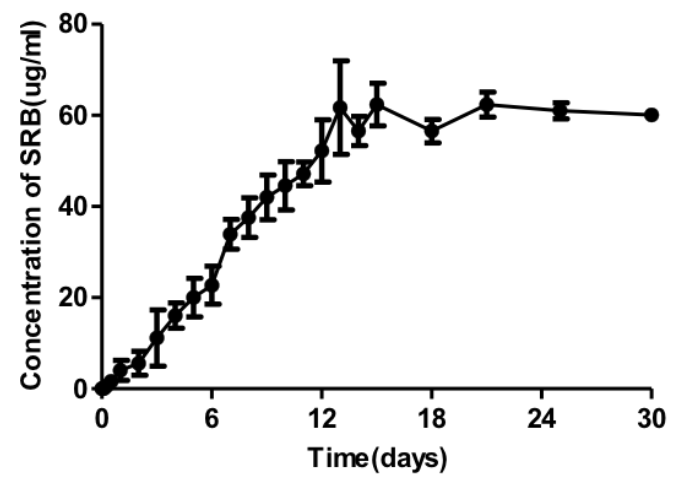

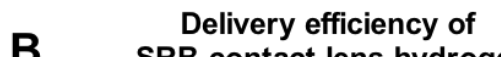

B SRB contact lens hydrogel

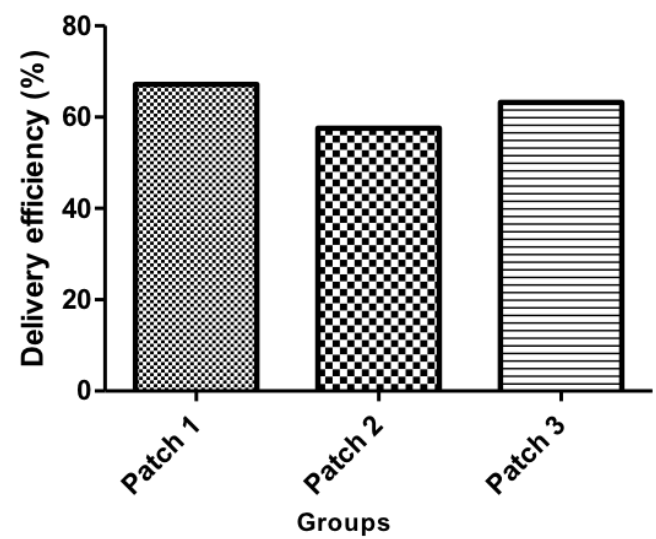

Figure 1. pHEMA hydrogel-based in vitro SRB delivery

A. Delivery kinetics of SRB. Dissected skin of BALB/c mice was exposed to $5 \mathrm{~mJ}$ laser treatment at 5\% coverage followed by topical application of pHEMA hydrogel 
patches. B. Patches were removed at day 30 and delivery efficiency was then quantified. $n=3$.

\section{pHEMA hydrogel-based in vivo SRB delivery}

Similar SRB patches were topically applied onto laser-treated mouse skin to study SRB delivery in vivo. We did not observe any change in color even at day 7 (Figure

2). We then removed hydrogel and found it completely dried, which could be the reason of inefficient delivery considering SRB could only diffuse out of hydrogel and through laser-generated skin MCs in an aqueous environment.
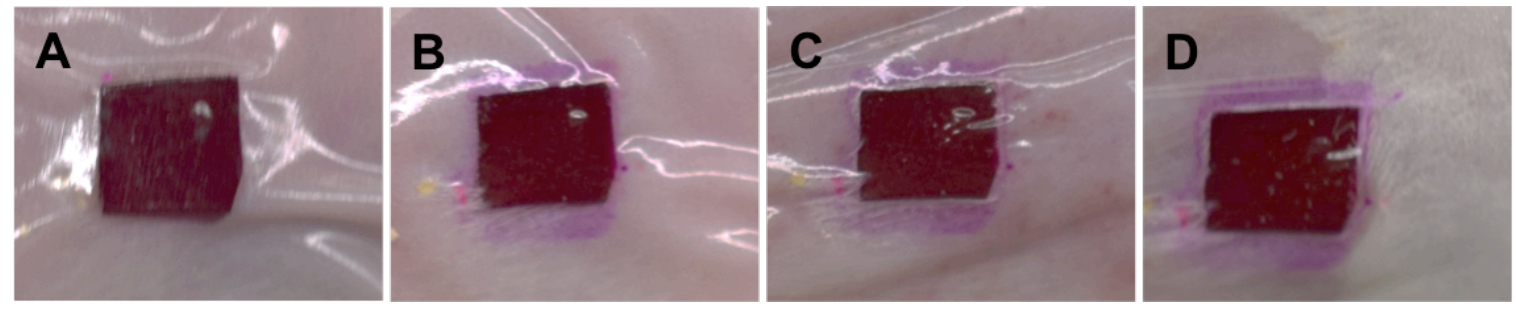

Figure 2. pHEMA hydrogel-based in vivo SRB delivery

Lateral back skin of BALB/c mice was exposed to $5 \mathrm{~mJ}$ laser treatment at $5 \%$ coverage followed by topical application of contact lens hydrogel patches. Patch images were taken at $0(2 A), 2(2 B), 4(2 C)$ and $7(2 D)$ days indicating no change in color intensity.

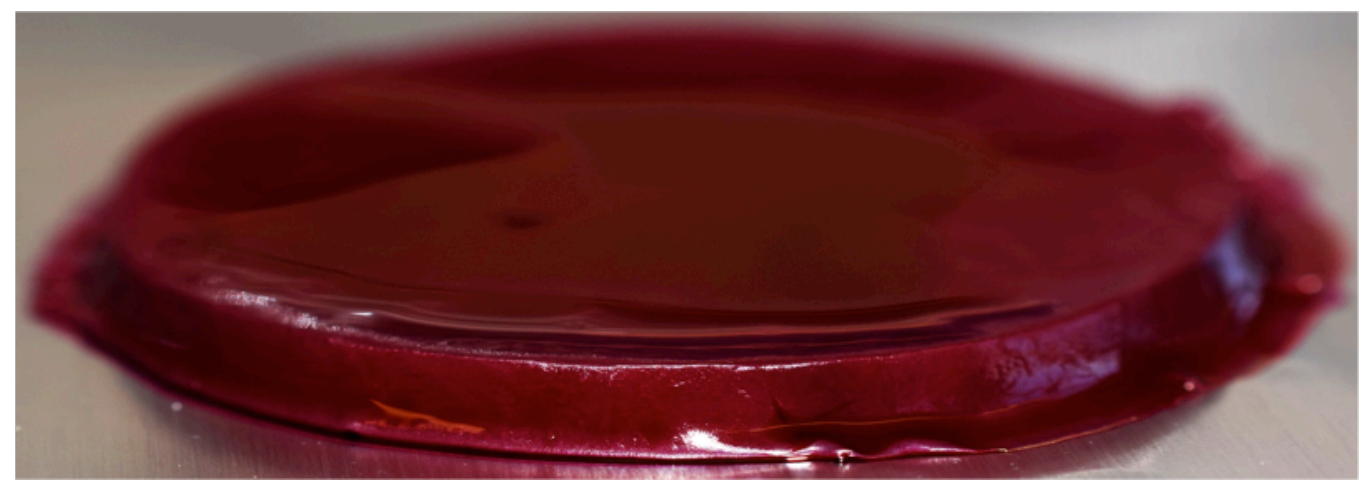

Figure 3. SRB-loaded PVA hydrogel 


\section{PVA hydrogel-based in vitro SRB delivery}

We then explored a water-rich PVA hydrogels for AFL-assisted drug delivery. To this end, we added SRB into 5\% w/v PVA solution followed by freeze-thaw cycles to yield relatively 'moist' hydrogels. A typical PVA-SRB patch is shown in figure 3. SRB loading amount was measured by extracting SRB from the hydrogel in PBS for 7 days with agitation on a regular basis until an almost clear hydrogel was visible. $1.8 \mathrm{mg}$ of SRB was loaded into $4 \times 4 \mathrm{~mm}^{2}$ patches with a thickness of $1.5 \mathrm{~mm}$ as shown in figure 3. Hairless mouse skin was dissected after laser treatment at $5 \mathrm{~mJ} / 5 \%$. The skin was placed on top of the receptor compartment of a Franz diffusion cell and secured with a $3 \mathrm{M}$ tegaderm film after patch application. The receptor compartment was filled with $1.5 \mathrm{ml}$ of PBS and $100 \mu \mathrm{l}$ samples were collected at different time points up to 10 days. As shown in Figure 4A, SRB permeation rose to around $150 \mu \mathrm{g} / \mathrm{ml}$ at day 10. Delivery efficiency was calculated for all the patches as shown in figure 4B.
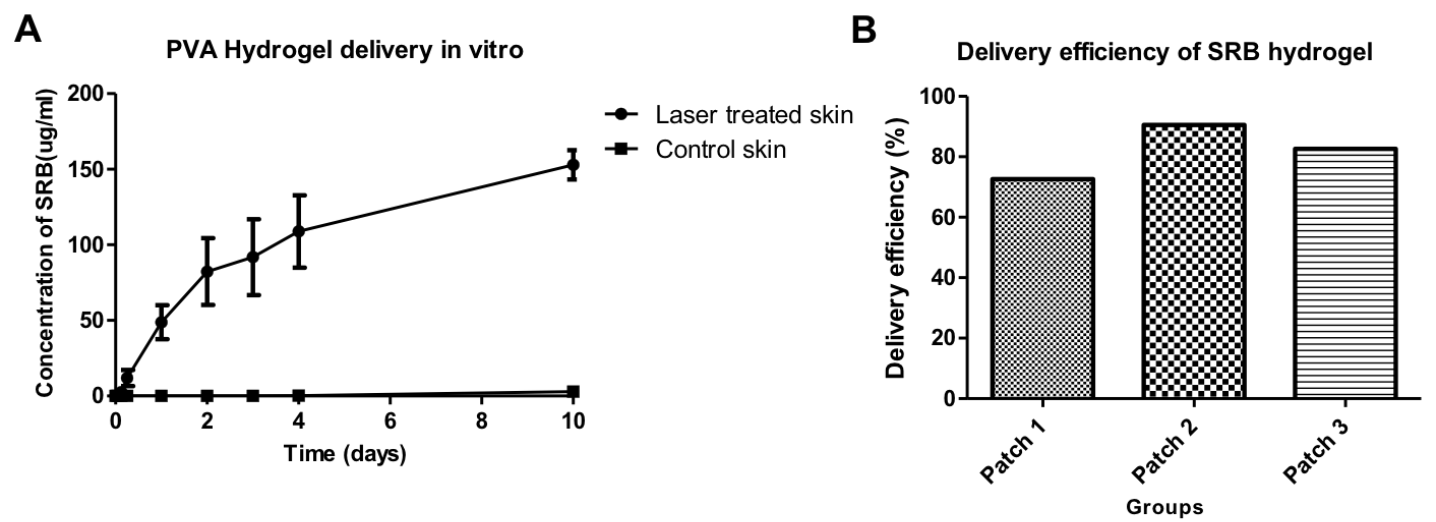

Figure 4. PVA hydrogel-based in vitro SRB delivery

A. Dissected skin of BALB/c mice was exposed to 5 mJ laser treatment at $5 \%$ coverage followed by topical application of PVA hydrogel patches containing SRB. Delivery kinetics were measured in comparison to untreated skin. B. Patches were removed day 10 and delivery efficiency was then quantified. $n=3$. 


\section{PVA hydrogel-based in vivo SRB delivery}

Next we explored whether laser-based PVA hydrogel could achieve high-efficient delivery of SRB in vivo. Mouse skin was treated with $10 \mathrm{~mJ} / 10 \%$ laser in a $4 \times 4 \mathrm{~mm}^{2}$ area. SRB patches with $2.8 \mathrm{mg}$ loading were used for this study. Patches were removed after 3 days when the color intensity of SRB looked faint by visual observation. The patches were extracted in PBS for seven days to quantify remaining SRB amount. Delivery efficiency was found to be approximately $99 \%$ at the end of the 3-day period (Figure 5).

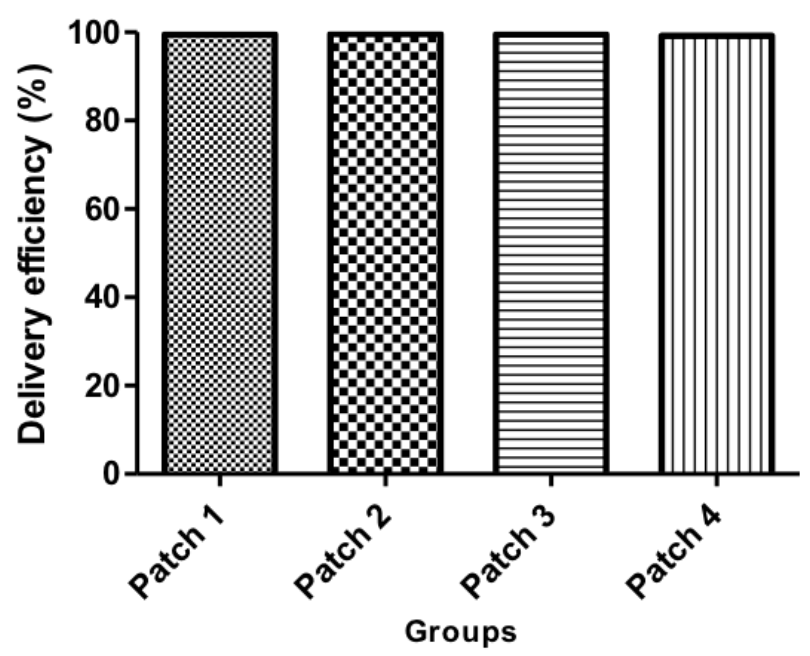

Figure 5. PVA hydrogel-based in vivo SRB delivery

Lateral back skin of BALB/c mice was exposed to $10 \mathrm{~mJ}$ laser treatment at $10 \%$ coverage followed by topical application of PVA-SRB hydrogel patches with $2.8 \mathrm{mg}$ SRB. Patches were removed day 3 and delivery efficiency was then quantified. $n=4$.

\section{PVA hydrogel-based in vivo SRB delivery at increased doses}

To test delivery capacity of the hydrogel system, we incorporated a higher dose of SRB by increasing the amount of SRB powder added to the PVA solution. Patch size 
and thickness remained same as in previous studies. Drug loading per patch was increased to $6.9 \mathrm{mg}$. We used the same laser condition of $10 \mathrm{~mJ} / 10 \%$ to observe the efficacy of delivery. Similar delivery efficiency of $99 \%$ was observed at the end of 3 days (Figure 6).

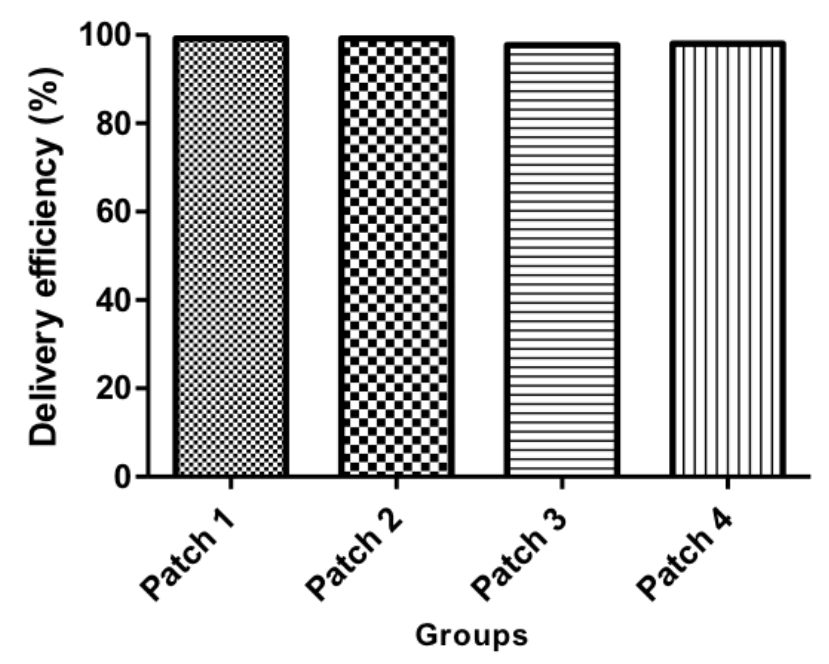

Figure 6. In vivo delivery of PVA-SRB hydrogels

Lateral back skin of BALB/c mice was exposed to $10 \mathrm{~mJ}$ laser treatment at $10 \%$ coverage followed by topical application of PVA-SRB hydrogel patches with $6.9 \mathrm{mg}$ SRB. Patches were removed 3 days later and delivery efficiency was then quantified. $n=4$.

\section{PVA hydrogel-based in vivo AZT delivery}

We found coating SRB into PVA hydrogels achieved more than $99 \%$ delivery efficiency at the end of 3 days. Considering this, we explored the delivery efficiency and kinetics of a real drug AZT. Approximately $1.8 \mathrm{mg}$ of drug was loaded into a $5 \times 5$ $\mathrm{mm}^{2}$ patch with a thickness of $2 \mathrm{~mm}$. A typical AZT hydrogel patch following freeze thaw cycle (before cutting into smaller patches) is shown in figure 7. Mouse skin was ablated with $10 \mathrm{~mJ} / 10 \%$ laser followed by patch application. Skin area of $5 \times 5 \mathrm{~mm}^{2}$ in 
female BALB/c mice was treated with laser and the patch was secured in place with a $3 \mathrm{M}$ tegaderm film and covered with bandage to prevent patch removal. Approximately $30 \mu \mathrm{l}$ of blood was collected at various time points and serum was separated and stored in $-80^{\circ} \mathrm{C}$ until quantification.

AZT amount in blood and remaining patches was quantified using LC-MS/MS method. We observed complete delivery of the drug within 24 hours with the drug concentrations peaking at 3 hours. Drug concentrations fell to baseline at around 15 hours (Figure 8A). Delivery efficiency was calculated for all patches after delivery and was found to be $100 \%$ (Figure $8 \mathrm{~B}$ ).

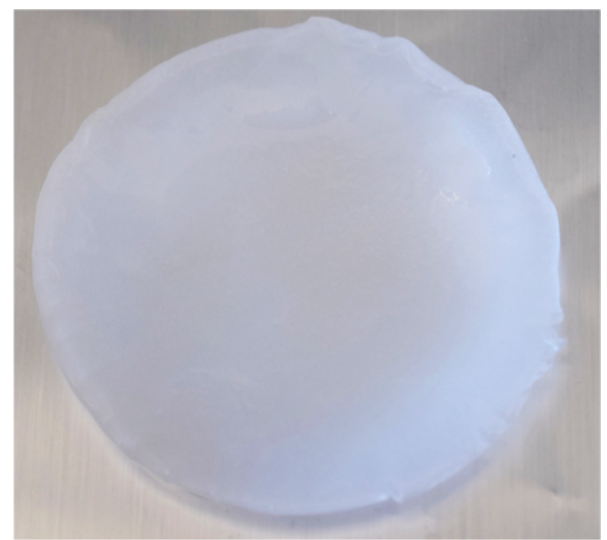

Figure 7. AZT loaded PVA hydrogel

AZT loaded PVA hydrogel, which is $2 \mathrm{~mm}$ in thickness. 
A

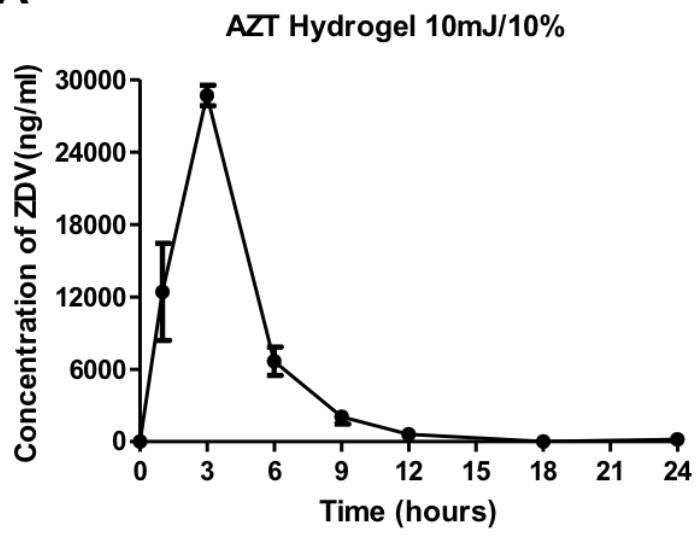

B

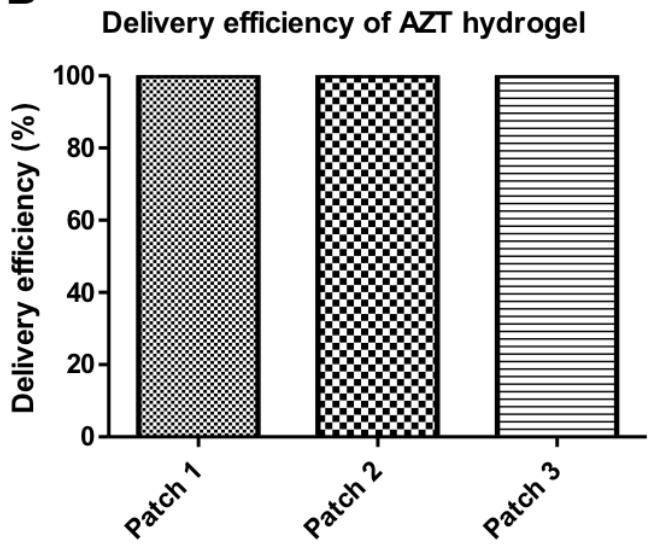

Figure 8. In vivo delivery of PVA-AZT hydrogels

A. Lateral back skin of BALB/c mice was exposed to $10 \mathrm{~mJ}$ laser treatment at $10 \%$ coverage followed by topical application of PVA-AZT hydrogel patches. Delivery kinetics were measured using LC-MS/MS. B. Patches were removed day 3 and delivery efficiency was then quantified. $n=3$. 


\section{Discussion}

In this study, we explored polymeric hydrogel-based drug delivery via AFL-generated skin MCs. pHEMA hydrogel elicited linear SRB release for 15 days (Figure 1A) and achieved a delivery efficiency of around 60\% (Figure 1B) in in vitro Franz cell systems. However, it failed to elicit high-efficient delivery in vivo potentially due to the challenge to maintain a moisturized system for hydrogel-based deliveries. This can be supported by the fact that the hydrogel dried up within 1-2 days of application to mouse skin and delivery could not be continued. This can also be due to the difference in water content in pHEMA and PVA hydrogels. Once the contact lens hydrogel is applied to laser-ablated skin, only one face of the patch is in contact with the transepidermal water. This water is not taken up by the hydrogel system due to drying of the polymer matrix. Hence, it is hard to maintain an environment suitable for drug dissolution via aqueous skin MCs.

We found high efficient delivery of SRB and AZT in vitro and in vivo for hydrogels formulated with high-molecular-weight PVA (Figures 4-7). More than $80 \%$ SRB was delivered in vitro at day 10 (Figure 4B). We further explored PVA hydrogel-based in vivo delivery of SRB at two loading levels (2.8mg and $6.9 \mathrm{mg})$. Complete delivery of SRB at the two loading levels was achieved in 3 days (Figure 5, 6). The reason of such high delivery efficiency can be attributed to the water-rich hydrogel itself, which facilitates easier and faster diffusion of drugs through skin MCs.

Lastly, we explored PVA hydrogel-based real drug AZT delivery via AFL-generated skin MCs in vivo. Serum AZT concentration peaked at 3 hours and returned to baseline at around 15 hours (Figure 7A). When compared to oral AZT delivery in 
Chapter I, which peaked at 30 minutes and returned to baseline at 3 hours, hydrogel mediated delivery was able to extend the duration of delivery by 5 folds. We found almost complete delivery of AZT at the end of 24 hours (Figure 7B). In our previous oral AZT administration (Figure 3D, Manuscript I), we obtained an area under the curve (AUC) of 8,666 ng.h/ml at $0.32 \mathrm{mg}$ dose. For AZT hydrogel delivery, AUC was calculated to be $119,868 \mathrm{ng} . \mathrm{h} / \mathrm{ml}$ at $1.8 \mathrm{mg}$ dose. We then compared the relative bioavailability of the two deliveries and found AFL-assisted hydrogel delivery increased AZT bioavailability by $\sim 130 \%$ as compared to oral delivery. The PVA hydrogel could hold approximately $1.8 \mathrm{mg}$ AZT and maintain its form when stored at $4^{\circ} \mathrm{C}$ in sealed bags. For a $10 \mathrm{~cm}^{2}$ patch, almost $72 \mathrm{mg}$ drug can be loaded for efficient delivery. Such a delivery capacity exceeds traditional patches, which contain a maximal drug dose of $\sim 10 \mathrm{mg}$.

To our knowledge, this is the first study to explore hydrogel-based drug delivery via laser-generated skin MCs. The safe laser technology and efficient delivery warrants further investigation for needle-free, painless transdermal drug and vaccine delivery.

\section{Conclusion}

In summary, we explored AFL-based hydrogels for small molecule drug delivery in murine models. PVA hydrogels were found as an ideal delivery platform for efficient and sustained delivery of SRB and AZT via laser-generated skin MCs. The convenient AFL-based hydrogel drug delivery warrants further investigation for potential clinical use. 


\section{Acknowledgements}

This work is partly supported by the National Institutes of Health grants DA033371 and AI107678 (to X.Y.C.). 


\section{References}

[1] Langer R. Drug delivery and targeting. Nature. 1998;392:5-10.

[2] Hoare TR, Kohane DS. Hydrogels in drug delivery: Progress and challenges. Polymer. 2008;49:1993-2007.

[3] Tiwari G, et al. Drug delivery systems: An updated review. Int J Pharm Investig. 2012;2:2-11.

[4] Tibbitt MW, Dahlman JE, Langer R. Emerging frontiers in drug delivery. J Am Chem Soc. 2016;138:704-717.

[5] Wenande, E., Olesen, U. H., Boesen, M. R., Persson, D. P., Lerche, C. M., Stürup, S., Gammelgaard, B., Husted, S., Anderson, R. R., ... Haedersdal, M. (2018). Laserassisted delivery enhances topical uptake of the anticancer agent cisplatin. Drug delivery, 25(1), 1877-1885.

[6] Yogeshwar G. Bachhav a, Arne Heinrich b , Yogeshvar N. Kalia. Using laser microporation to improve transdermal delivery of diclofenac: Increasing bioavailability and the range of therapeutic applications. European Journal of Pharmaceutics and Biopharmaceutics 78 (2011) 408-414.

[7] E.-H. Lim, H. Kim, Y.-O. Park, et al. Toenail onychomycosis treated with a fractional carbon-dioxide laser and topical antifungal cream. J Am Acad Dermatol, 70 (5) (2014), pp. 918-923.

[8] Y. Cao, P. Kakar, M.N. Hossen, M.X. Wu, X. Chen. Sustained epidermal powder drug delivery via skin microchannels. J. Control. Release, 249 (2017), pp. 94-102. 
[9] Bodugoz-Senturk H, Macias CE, Kung JH, Muratoglu OK. Poly(vinyl alcohol)acrylamide hydrogels as load-bearing cartilage substitute. Biomaterials. 2009;30:589596.

[10] Thorn R, Greeman J, Austin A. An in vitro study of antimicrobial activity and efficacy of iodine-generating hydrogel dressings. J Wound Care. 2006;15:305.

[11] Momoh FU, Boateng JS, Richardson SC, Chowdhry BZ, Mitchell JC.

Development and functional characterization of alginate dressing as potential protein delivery system for wound healing. Int J Biol Macromolec. 2015;81:137-150.

[12] Pandit A, Ashar R, Feldman D. The effect of TGF- $\beta$ delivered through a collagen scaffold on wound healing. J Invest Surg. 1999;12:89-100.

[13] Jayakumar R, Prabaharan M, Kumar PS, Nair S, Tamura H. Biomaterials based on chitin and chitosan in wound dressing applications. Biotechnol Adv. 2011;29:322337.

[14] Ishii S, Kaneko J, Nagasaki Y. Development of a long-acting, protein-loaded, redox-active, injectable gel formed by a polyion complex for local protein therapeutics. Biomaterials. 2016;84:210-218.

[15] Liu W, Griffith M, Fengfu L. Alginate microsphere-collagen composite hydrogel for ocular drug delivery and implantation. J Mater Sci Mater Med. 2008;19:33653371.

[16] Dash A, Cudworth G. Therapeutic applications of implantable drug delivery systems. J Pharmacol Toxicol Methods. 1998;40:1-12.

[17] Yu L, Ding J. Injectable hydrogels as unique biomedical materials. Chem Soc Rev. 2008;37:1473-1481. 
[18] García-Millán, Koprivnik, and Otero-Espinar. "Drug Loading Optimization and Extended Drug Delivery of Corticoids from PHEMA Based Soft Contact Lenses Hydrogels via Chemical and Microstructural Modifications." International Journal of Pharmaceutics 487.1-2 (2015): 260-69.

[19] J.E. Rower, B. Klein, L.R. Bushman, P.L. Anderson, Validation of a sensitive LC/MS/MS method for the determination of zidovudine and lamivudine in human plasma, Biomed. Chromatogr. 26 (2012) 12-20.

[20] Prausnitz, M. R., \& Langer, R. (2008). Transdermal drug delivery. Nature biotechnology, 26(11), 1261-8. 